Review

\title{
Effects of Quantum Confinement of Hydrogen in Nanocavities - Experimental INS Results and New Insights
}

C. Aris Chatzidimitriou-Dreismann *

Institute of Chemistry, Secr. C2, Faculty II, Technical University of Berlin, D-10623 Berlin, Germany; EMail: chariton.dreismann@tu-berlin.de

* Correspondence: C. Aris Chatzidimitriou-Dreismann; E-Mail: chariton.dreismann@tu-berlin.de

Academic Editor: Sotirios Baskoutas

Special Issue: Quantum Confinement Effects in Nano Material

Recent Progress in Materials Received: March 21, 2020

2020, volume 2, issue 2

Accepted: May 29, 2020

doi:10.21926/rpm.2002015

Published: June 23, 2020

\begin{abstract}
Current developments of non-relativistic quantum mechanics appear to predict, and reveal, counter-intuitive dynamical effects of hydrogen in nanostructured materials that are of considerable importance for basic research as well as for technological applications. In this review, the experimental focus is on $\mathrm{H}_{2} \mathrm{O}$ and $\mathrm{H}_{2}$ molecules in carbon nanotubes and other nanocavities that have been experimentally investigated using the well-established technique of incoherent inelastic neutron scattering (INS). For instance, the momentum and energy transfers, as obtained from the commonly used standard data analysis techniques, from a

(I) $\mathrm{H}_{2}$ molecule in a $\mathrm{C}$-nanotube resulting in a roto-translational motion along the nanotube axis

seems to (1) either violate the standard conservation laws or (2) to attribute to the $\mathrm{H}_{2}$ molecule undergoing translation the effective mass $M \approx 0.64$ a.m.u. (atomic mass units) instead of the expected 2 a.m.u. A similar striking anomalous effect has been found in the neutron- $\mathrm{H}$ scattering from the
\end{abstract}

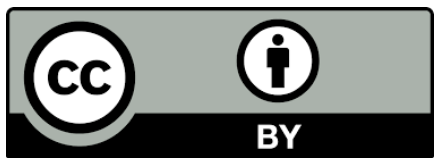

(C) 2020 by the author. This is an open access article distributed under the conditions of the Creative Commons by Attribution License, which permits unrestricted use, distribution, and reproduction in any medium or format, provided the original work is correctly cited. 
(II) $\mathrm{H}_{2} \mathrm{O}$ molecules in nano-channels of some solid materials, in which $\mathrm{O}-\mathrm{H}$ stretching vibrations along the channel axis are created.

The results of this scattering process seem to once again either violate the standard conservation laws or to attribute to the effective mass of the struck $\mathrm{H}_{2}$ molecule as $M \approx 0.55$ a.m.u. instead of the expected value of 1 a.m.u. We show that these counterintuitive observations from the INS studies have no conventional interpretation within the standard nonrelativistic scattering theory. However, they can be qualitatively interpreted "from first principles" within the framework of modern theories of

(III) time-symmetric quantum dynamics, as provided by the weak values (WV) and two-statevector formalism (TSVF),

and/or

(IV) quantum correlations, especially quantum discord (QD) and quantum thermodynamics (QTD).

The theoretical analysis provides an intuitive understanding of the experimental results, gives strong evidence that the nano-structured cavities do represent quantum systems which participate significantly in the dynamics of the neutron-H scattering, and, surprisingly, shows that new physical information can be derived from the experimental data. This latter point may also have far-reaching consequences for technology and material sciences (e.g., fuel cells, $\mathrm{H}$ storage materials, etc.). Moreover, novel insights into the short-lived quantum dynamics and/or quantum information theory can be gained.

\section{Keywords}

Quantum dynamical effects of confined hydrogen; hydrogen-nanomaterial interactions; quantum discord; entanglement; quantum Maxwell's demon; negative conditional entropy; energy-costs of correlations; weak values; two-state-vector formalism; inelastic incoherent neutron scattering

\section{Introduction}

Recent theoretical progress in (non-relativistic) quantum mechanics has provided novel insights into the various physical and technological fields and has also allowed us to predict new effects and to conceive new technological applications. In the first (i.e., experimental) part of this review, we will consider a new quantum effect of scattering accompanying an elementary collision of two quantum systems, namely a neutron and a proton (or $\mathrm{H}$ atom), in which the latter interacts with an environment, with $\mathcal{E}$, being of quantum nature. In clear contrast to a classical environment, we will see that the presence of a quantum $\mathcal{E}$ can cause counterintuitive effects of momentum- and energytransfers which contradict the conventional expectations. Experimental evidence of a recently discovered "anomalous" effect, namely, the deficit of momentum transfer (equivalently, reduced effective mass of the scatterer) in a neutron-atom collision, has been presented and discussed in 
some detail. In an example considered in the following Section, the technique of non-relativistic incoherent inelastic neutron scattering (INS) of thermal neutrons has been applied on single $\mathrm{H}_{2}$ molecules confined in multi-walled carbon nanotube channels and other related materials. On interpreting the results within the conventional theory, the experimental findings reveal a significantly reduced effective mass of the recoiling $\mathrm{H}_{2}$ molecule, of $M=0.64$ a.m.u. (atomic mass units), which of course is unrealistic.

As we will discuss in the following sections, the epithet "anomalous" refers to the expectations of the conventional theory [1]. To put it in more illustrative terms: the struck particles (molecules, atoms) seem to be more mobile than even the free particles. This, of course, cannot have any interpretation in the frame of conventional theories. To demonstrate the specific reason for the failure of the conventional theoretical viewpoint, it will be necessary to consider, in detail, several crucial experimental details of the neutron scattering spectrometer and the measuring procedure. Here, it is essential to be aware of the following questions: What, in fact, is exactly measured? In addition, what is inferred from the measured data and how? The answers to these questions will be given in Section 2 , which constitutes the Experimental Part of the present review article.

The aforementioned "anomalous" effect is explored and interpreted "from first principles" in the following two Theoretical Parts (see Sections 3 and 4). Here, the experimental results find a straightforward qualitative interpretation within modern quantum mechanics and quantum information theory. We discuss two such actual theoretical frameworks, which, although seemingly quite independent from each other, succeed in interpreting the measured data as well as reveal the novel physical aspects of the phenomenon.

The first theoretical framework (Section 3) is given by the modern theory of WV and TSVF of Aharonov and collaborators, the development of which started several decades ago. This novel theory gives us an understanding of the scattering phenomenon under consideration based on a quantum mechanism.

The second one (Section 4) is based on the recent progress in quantum thermodynamics and the associated quantumness of correlations (especially quantum discord, discovered in 2001 independently by Vedral and Zurek). This gives us an understanding based on quantum correlations. The following additional introductory remarks should be in order.

I. On quantum mechanisms - A fundamental feature of non-relativistic quantum mechanics, captured in the time-inversion symmetry of Schrödinger's equation, was shown to play a crucial role in the novel theory of WV and TSVF (see, e.g., [2-9] and references cited therein). Various novel quantum effects were predicted, and new experiments were proposed and carried out, which confirmed the theoretical predictions (see, e.g., [10-12] and references therein). Surprisingly, the physical reality of the wavefunction was also further exposed and clarified by giving an experimental procedure to measure the real and imaginary part of the wavefunction [11]. However, in the present article, the focus is mainly on the applicability of the general WV-TSVF theory to real scattering experiments, rather than on purely theoretical or interpretational issues. The experimental momentum-transfer deficit under consideration has been recently investigated and interpreted by Chatzidimitriou-Dreismann [13-15]. 
The theories of WV and TSVF are nowadays a well established research field, providing new physical insights and novel experimental techniques. In the present review article, a new class of experiments related to momentum and/or energy transfer have been discussed, with the aim of strengthening the connection of the "theory"-community with that of the experiments conducted in the field of scattering physics (cf. in particular [15]).

II. On quantum correlations - Quantum correlations between electrons, resulting due to the indistinguishability of identical fermions, are already well known in traditional quantum physics and chemistry of atoms, molecules, and solids. In contrast, quantum correlations between non-identical particles (e.g., electrons and nuclei) are almost unknown in many fields, such as chemistry and material sciences. Another research field, in which such quantum correlations are assumed to play no role, is the conventional and widely applied neutron scattering theory (see, e.g., the textbook [1]). Although a neutron is a quantum object and thus, in general, should become entangled with a scattering nucleus, this characteristic quantum feature is absent in the standard theory. Here, the quantum degrees-of-freedom of the neutron do not appear in any basic scattering expression (e.g., the equation for the partial differential cross sections, which are the directly measurable quantities) only $c$-numbers which refer to a neutron appear in these formulae, e.g., the cross sections (or the scattering lengths) of the neutron-nucleus collision.

The new experimental findings discussed below may be considered to be caused by such quantum correlations, thus also providing a new operational meaning to the various proposed theoretical measures (or quantifiers) of the so-called quantumness of correlations, such as entanglement and quantum discord. As we will show in the following sections, the presented experimental findings are often counterintuitive and cannot have a conventional interpretation, if the usual standard analysis of the measured data is applied.

More generally, the second theoretical part (Section 4) uses concepts of quantum information [16] and non-classical correlations. Our analysis touches upon investigations of quantum entanglement and, in particular, with its extension and/or generalization within the theoretical framework, broadly known as the quantumness of correlations (QoC); corresponding studies started in 2001 [17, 18]. In particular, the focus is on experimentally accessible thermodynamic and/or energetic consequences of quantum correlations, such as the work costs for the erasure of information, which sometimes are predicted to even be "negative" [19]. The field of QoC is alread huge (see, e.g., the review article by Modi et al. [20]). However, until now, it has remained rather unnoticed in most experimental physical (perhaps with the exception of photonics) and other scientific fields.

The main concepts in this paper have been presented at the interdisciplinary COST-Action “Nanoscale Quantum Optics International Workshop, Prague, Feb. 13-16, 2018”.

Full details of the theoretical derivations and additional experimental results were reported in the original Refs. [13] and [21], on which the present review article is also based.

\section{Experimental Part - INS from Protons in Nanocavities}


To facilitate the motivation of the present investigations, we start with the presentation of a striking experimental effect under consideration.

To prevent a possible source of confusion, a note on the common scientific nomenclature is in order here:

(1) In this paper, only non-relativistic quantum mechanics is considered.

(2) The neutron-nucleus (or neutron-atom) collision is called elastic since the internal degrees-offreedom of the colliding particles do not play a role in the dynamics of the reaction (i.e., the total kinetic energy of the two particles remains constant).

(3) The experimental technique of INS contains the epithet inelastic, which simply indicates that the neutron loses some kinetic energy in the collision (which, of course, is gained by the scattering system).

INS from protons is, for a large part, incoherent due to the strong spin-flip process accompanying the collision (cf. [1]). As nicely explained by Feynman [22], this spin-flip is tantamount to the gained path information that prevents quantum interference between partial scattering waves originating from different scattering particles. In other words, in the case of INS from hydrogen, one can say that "each neutron represented by a measured intensity peak has been scattered by one proton only".

In this paper, we mainly consider isotropic scattering, which is also the case for incoherent scattering of thermal neutrons from $\mathrm{H}$. Due to the low kinetic energy of these neutrons, the process belongs to the non-relativistic regime [1].

\subsection{An Introductory Example: Neutron Scattering from Liquid He}

For illustrating the expectations of the conventional INS theory [23, 24] (and, in general, of the conventional theory of thermal neutron scattering [1]), let us first present the results of neutron scattering from liquid $\mathrm{He}$, which also obeys the rules of the conventional INS.

Here, the validity of the conventional energy and momentum conservation is clearly demonstrated (see Figure 1). The red line represents the so-called free recoil parabola of the He atom in the $K-E$ plane (often called the momentum-energy plane) and is given by

$$
E=\frac{(\hbar K)^{2}}{2 M}
$$

where $\mathrm{M}$ is the mass of the ${ }^{4} \mathrm{He}$ atom, $\hbar K$ is the momentum transfer from the neutron $(n)$ to the He atom, $E$ is the energy transfer from $n$ to $\mathrm{He}$. The parabola fits the measured data (blue points) excellently. This parabola represents the well-known impulse approximation $[1,27,28]$ for a twobody collision of the standard theory. Thus, the validity of the conventional theoretical prediction is confirmed. The original high-precision measurements [25] were recently carried out using the wide angular-range chopper spectrometer (ARCS) [26]. Obviously, the experimental results are in agreement with the conventional theoretical expectations (see Eq. (12) below). 


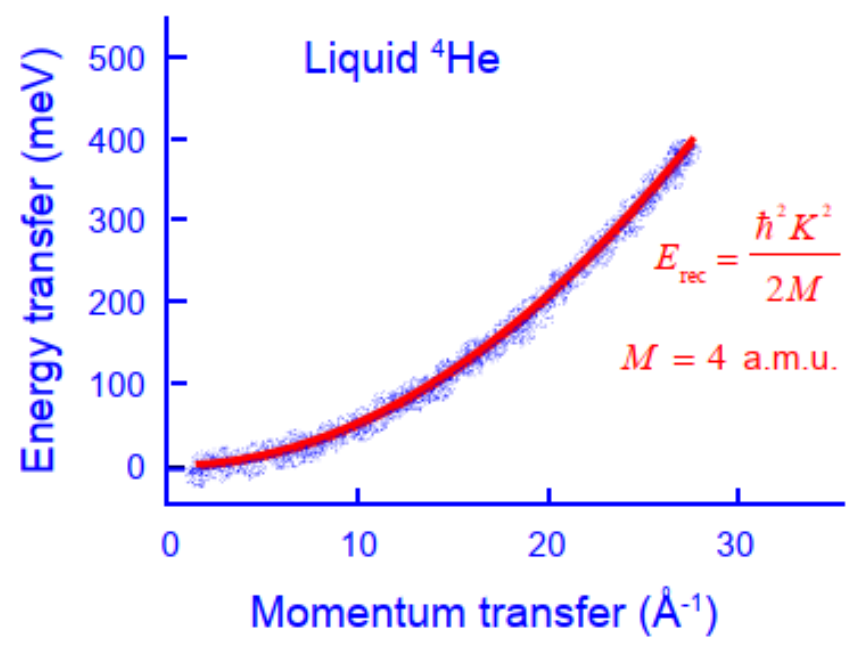

Figure 1 Graphical representation of the measured dynamic structure factor, $S(K, E)$, of liquid helium [25]. The red line is the calculated recoil parabola (see Eq. (1)) with the mass of ${ }^{4} \mathrm{He}$ equal to $M=4$ a.m.u. The white-blue points around the recoil parabola are data measured with the time-of-flight (TOF) instrument ARCS [26]. (Taken from Ref. [15] with permission.)

\subsection{Details of the Scattering Spectrometer - What is Directly Measured}

The experimental technique available at pulsed neutron source facilities, which are the most upto-date neutron facilities, is the time-of-flight (TOF). The experimental results considered in this paper have been measured employing this method.

An outline of a standard TOF scattering experiment is as follows (cf. Figure 2). A short pulse of neutrons produced by the spallation source reaches the monitor (i.e., the clock that starts the TOF measurement). $L_{0}$ is the distance between the monitor and the sample. A neutron of the pulse, which scatters from the sample and reaches the detector, triggering the stop signal of the TOF measurement. $L_{1}^{\theta}$ (or $L_{1}$, for simplicity) is the sample-detector distance and the corresponding scattering angle is $\theta$.

For a measured TOF value, $t_{\text {TOF }}$, holds

$$
t_{T O F}=\frac{L_{0}}{v_{0}}+\frac{L_{1}^{\theta}}{v_{1}}+t_{0}
$$

where $v_{0}$ and $v_{1}$ are the velocities of the neutron before and after scattering, respectively and $t_{0}$ is a time-offset parameter caused due to the electronics. When $v_{0}$ (and equivalently, the initial kinetic energy $E_{0}$ ) have experimentally predetermined fixed values, one has a "direct geometry TOFspectrometer". ARCS, SEQUOIA and MARI, which are mentioned in the present paper, belong to this class of spectrometers. 


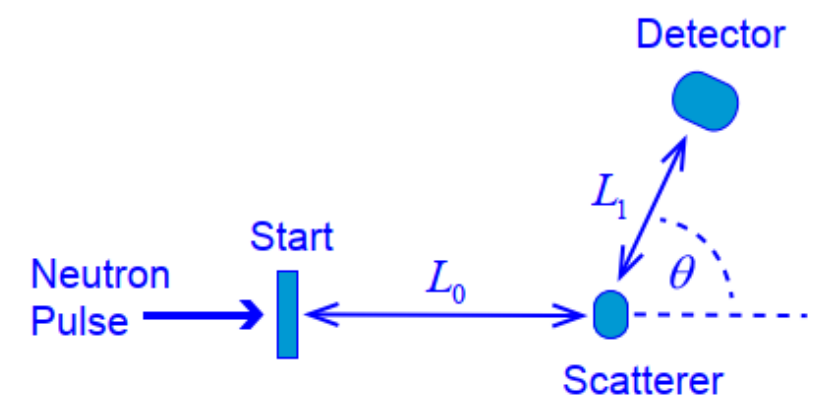

Figure 2 Schematic of a time-of-flight (TOF) scattering experiment.

The scattering intensity measured by the detector is a function of the energy loss $E$ of the neutron

$$
\begin{aligned}
E & =E_{0}-E_{1}=\frac{1}{2} m v_{0}^{2}-\frac{1}{2} m v_{1}^{2} \\
& =\hbar \omega=\frac{\left(\hbar k_{0}\right)^{2}}{2 m}-\frac{\left(\hbar k_{1}\right)^{2}}{2 m}
\end{aligned}
$$

where $m$ : is the neutron mass. The corresponding momentum $\hbar \mathbf{K}_{n}$ transferred on the neutron due to the two-body scattering is

$$
\hbar \mathbf{K}_{n}=\hbar \mathbf{k}_{1}-\hbar \mathbf{k}_{0}
$$

It holds that

$$
\left|\mathbf{K}_{n}\right|=K_{n}=\sqrt{k_{0}^{2}+k_{1}^{2}-2 k_{0} k_{1} \cos \theta}
$$

Here, the subscripts 0 and 1 refer to the values before and after the neutron-atom impulsive collision, respectively. Since we consider an incoherent [1,22] scattering, the exchange of momentum and energy is between a neutron and (the nucleus of) a single atom [1, 22]. Moreover, due to Newton's third law of "action = reaction", the struck atom (nucleus) receives the momentum transfer $\mathbf{K}_{A}$ from the neutron. Thus,

$$
\mathbf{K}_{A}=-\mathbf{K}_{n}
$$

and one defines

$$
K \equiv\left|\mathbf{K}_{A}\right|=\left|\mathbf{K}_{n}\right|
$$

If the sample is at rest, the neutron scatters from a free atom with mass $M$ and zero mean initial momentum, $\langle P\rangle=0$. Here, due to the conservation of kinetic energy and momentum, the simple kinematic relation (see, e.g., [28]) holds.

$$
\frac{k_{1}}{k_{0}}=\frac{\cos \theta+\sqrt{(M / m)^{2}-\sin ^{2} \theta}}{\frac{M}{m}+1}
$$

This relation holds for the centroid of the measured intensity peak, which has a finite width due to the well known Doppler effect [1], as well as a small contribution due to the limitation of the 
instrument's resolution. Parenthetically, the shape analysis and theoretical interpretation of a measured intensity peak constitute a predominant part of many neutron scattering investigations.

As several experimental details play a crucial role in the theoretical framework under consideration, the following facts should be pointed out:

(a) From the measured TOF value (2), expressed by Eq. (2), the value of $v_{1}$ is obtained. Similarly, the values of $k_{1}=\left|\mathbf{k}_{1}\right|$, since $m v_{1}=\hbar k_{1}$; and consequently of the energy transfer, $E=\hbar \omega$, (see Eq. (3)) are obtained. It should be noted that the value of $\theta$ does not play any role here. We stress that the numerical value of $E$ for every single elementary scattering process is independent of any tacit theoretical beliefs or assumptions since it immediately follows from the actually measured TOF value.

(b) The momentum transfer $|\hbar \mathbf{K}|$, Eq. (5), is estimated from both the measured TOF value and the direction given by $\theta$ in which the detector is positioned. Moreover, Eq. (8) connects the values of $\theta$, which is an instrumental parameter, and the atomic mass $M$. Usually, one tacitly assumes that the atomic mass $M$ is well known (recall that non-relativistic quantum mechanics holds here) and thus the ratio $\frac{k_{1}}{k_{0}}$ is fully determined by $M$ and $\theta$.

Hence one sees the following crucial point: If the scattering particle, due to the interactions with its environment, becomes "dressed" with environmental degrees of freedom, then the numerical value of $M$ appearing in the above kinetic equations will not coincide with that of the free particle. As a matter of fact, it is quite often a common practice (or a usual procedure) to use the free-mass value of $M$ in order to "fine-tune" and/or "improve" the calibration of the instrumental set-up, i.e., to "optimize" the instrumental value of $\theta$ (and sometimes also of $L_{1}$ ). [The usual justification for doing this is that the impulsive neutron-nucleus interaction is very fast, due to the small range of the Fermi pseudo-potential of the strong force, and thus a neutron only collides with a nucleus which is "effectively free".] Obviously, this procedure is based on the belief that the simple (and strict) twobody elastic collision is a sufficient approximation here and thus the free-atom mass is the correct quantity for the parameter $M$ entering the above kinematic Eqs. (1-8).

To summarize, from each $t_{\text {TOF }}$ value measured with the detector in the direction $\theta$, the corresponding momentum $(\hbar K)$ and energy $(E=\hbar \omega)$ transfer from the neutron to the scattering particle (nucleus) are uniquely determined. Thus, we realize that each specific detector measures only one specific trajectory in the two-dimensional $K-E$ plane.

It should be noted that in the neutron scattering experiments from condensed systems, the recoiling particles of the sample cannot be measured. This is in contrast to other experimental fields studying scattering physics (e.g., high-energy physics), in which appropriate detector systems measure a great number of particles participating in a (usually relativistic) collisional process.

The scattering process produces an outgoing three-dimensional wave for the entangled atomneutron system, which for the amorphous samples is isotropic since the de Broglie wavelength of the neutron is much larger than the diameter of the nucleus [1]. This process is called the $s$-wave scattering. According to Eqs. (3) and (5), the instrumental parameters of the detector shown in Eq. (2) (denoted here by $\{\mathrm{IP}\}$ ) determine the corresponding scattering intensity, $I(\mathrm{~K}, E)$, and the corresponding dynamical structure factor, $S(\mathrm{~K}, \omega)[1]$, of the scattering probe, i.e.,

$$
I(\mathrm{TOF},\{\mathrm{IP}\}) \Rightarrow I(\mathrm{~K}, E) \Rightarrow S(\mathrm{~K}, \omega)
$$


Note that neutron spectrometers usually have many detectors at various angles $\theta$ for measuring the TOF values in the predetermined $K-E$ range of interest.

\subsubsection{Two-Body Collisions - Momentum and Energy Conservation}

We consider here the neutron-atom collision mentioned above in some more detail. The initial momentum, $\mathbf{P}$, of an atom, which in general is not zero, is related with the energy transfer, $E \equiv \hbar \omega$, from the neutron to the atom:

$$
\begin{aligned}
E=E_{0}-E_{1}=\hbar \omega & =\frac{(\hbar \mathbf{K}+\mathbf{P})^{2}}{2 M}-\frac{P^{2}}{2 M} \\
& =\frac{(\hbar K)^{2}}{2 M}+\frac{\hbar \mathbf{K} \cdot \mathbf{P}}{M}
\end{aligned}
$$

This equation represents energy conservation. $\hbar \mathbf{K}$ is the momentum transfer from the neutron to the atom, as has already been mentioned above.

The first term on the right-hand side of this equation is often called the recoil energy,

$$
E_{\text {rec }}=\hbar \omega_{\text {rec }}=\frac{(\hbar K)^{2}}{2 M}
$$

It represents the kinetic energy gained by a struck atom that was initially at rest. Therefore, since we have $\langle P\rangle=0$, we obtain the following relation for the involved mean values:

$$
\langle E\rangle=\frac{\hbar^{2}\langle K\rangle^{2}}{2 M} \equiv \bar{E}_{r e c}
$$

In simple terms, this equation refers to the centroid of the peak seen in the energy spectrum. Hence, INS from a gaseous sample of such atoms leads to an intensity peak centered at a transferred

energy value of $\bar{E}_{\text {rec }}$, and having a finite width caused due to the additional term $\hbar \mathbf{K} \cdot \frac{\mathbf{P}}{M}$. This term represents the aforementioned Doppler broadening. The centroid and width of the peak are shown in Figure 1, in which the data of the incoherent scattering from ${ }^{4} \mathrm{He}$ atoms in the liquid phase is shown (for details, see the original work [25]).

\subsection{INS and Vibrational Spectroscopy of Molecules}

Modern INS instruments have detector systems with a large number (e.g., about $10^{5}$ ) of individual detector pixels, which cover a large area of the two-dimensional $K-E$ plane on which the INS intensity map, or equivalently the dynamic structure factor, $S(K, E)$, are defined (cf. relations (9)). A key advantage of INS over the various standard optical methods (such as IR-absorption or Raman spectroscopy) is that the neutron, having a finite mass, causes significant transfer of energy and momentum to the struck atom. Thus, INS is intrinsically a "two-dimensional" spectroscopic method, which enables the extraction of potentially more, and even qualitatively new, dynamical information from the system under investigation.

In subsections 2.4 and 2.5, following the work described in the original paper [21], we report the results of some recent INS experiments investigating molecular vibrations. The first is INS from ice at $T=20 \mathrm{~K}$, which yields results that are fully in line with the conventional theoretical expectations. The second is INS from single $\mathrm{H}_{2} \mathrm{O}$ molecules in oriented nanoscopic channels of two solid crystalline 
materials called beryl and cordierite (see below). The results of this experiment contradict the conventional expectations and reveal the novel effect mentioned in the introduction. To see this, the measured data from the $\mathrm{OH}$-stretching vibrational modes have been compared. Moreover, another INS experiment from a single $\mathrm{H}_{2} \mathrm{O}$ molecules adsorbed on nanoporous $\gamma$-alumina has been observed to exhibit the same effect in the bending molecular vibration. This is in contrast to the conventional behavior of the bending $\mathrm{H}_{2} \mathrm{O}$ vibration in beryl and in bulk ice.

In the conventional INS vibrational spectroscopy, the concept of the Debye-Waller factor plays a significant role (cf. e.g., $[1,23])$. This factor, appearing in the expression of the scattering intensity, is $K$-dependent and thus causes an asymmetry of the vibrational peaks and the temperaturedependent $K$-shifts of the peak. This factor, however, does not affect the main experimental results of this Section, because here we compare the $K$-positions of the vibrational peaks of $\mathrm{H}_{2} \mathrm{O}$ molecules in different environments, but at similarly low temperatures. The latter implies that the Debye-Waller factor, as the aforementioned "anomalous" $\hbar K$-deficit is concerned, is of minor importance for the mentioned comparison.

\subsection{INS from Bulk Ice-Ih}

INS measurements from $\mathrm{H}$-containing samples are usually carried out at low temperatures since this lowers the "background" and also minimizes the effect of the Debye-Waller-factor [1] on $S(K, E)$, which is the quantity of primary interest for testing theoretical predictions.

Water in its solid or liquid phase has been studied extensively in the scientific literature of INS. In Figure 3 is presented the observed $S(K, E)$ of microcrystalline hexagonal ice (ice $\mathrm{lh}$ ) at about $T=20$ $\mathrm{K}$ and pressure of 1 bar. The initial neutron energy was $E_{0}=750 \mathrm{meV}$. The experiment was carried out using the TOF-spectrometer MARI of ISIS spallation source, UK [29]. The predominant parts of the scattering contributions are caused by $\mathrm{H}$ due to its large incoherent scattering cross section [1]. The shown black parabola line is the theoretical recoil line of a free $\mathrm{H}$ atom being initially at rest

$$
E_{\text {rec }}^{\text {free }}=\frac{\hbar^{2} K^{2}}{2 M_{H}}
$$

where $M_{H}$ is the atomic mass of $\mathrm{H}$. Note that the masses of neutron and $\mathrm{H}$ are almost equal, $m_{n} \approx M_{H}$. Clearly, this is equivalent to the preceding Eq. (12).

The intensity peak around $E=420 \mathrm{meV}$ and $K=14 \AA^{-1}$ represents the molecular stretching vibrational modes of $\mathrm{H}_{2} \mathrm{O}$. These have already been well studied with conventional IR and Raman spectroscopy $[23,24]$. One observes here the conventionally expected peak position, i.e., the centroid of this intensity peak overlaps with the theoretical free $\mathrm{H}$ atom recoil line.

In view of Eq. (13), the experimental result shows that the observed intensity peak is satisfactorily described with the common theoretical assumption that the collision is impulsive and effectively a two-body neutron-H scattering process. In other words, there is neither significant free recoil of the molecule as a whole, nor "dressing" of $\mathrm{H}$ with intramolecular and/or environmental degrees of freedom. Therefore, the effective mass of the scatterer is $M_{H}^{e f f} \approx 1.0$ a.m.u.

Moreover, the following point made by Kearley et al. Ref. [30] provides additional support to the above physical picture. In particular, the very short (i.e., within a time duration from picoseconds to 
femtoseconds) characteristic neutron-proton scattering time in INS, in connection with the significant spectral separation of the stretching from other modes, is expected to cause a decoupling of the "fast" $\mathrm{H}$-stretching vibrational motion from the other (slower) $\mathrm{H}$ motions.

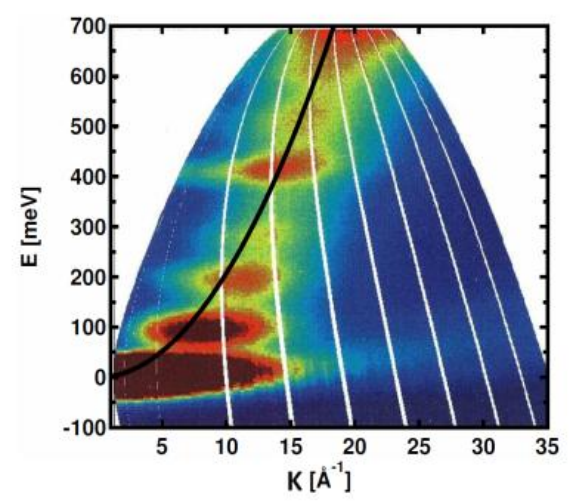

Figure 3 Measured $S(K, E)$ intensity map of ice $\mathrm{lh}$ at $20 \mathrm{~K}$ and $1 \mathrm{bar}$, recorded using the MARI spectrometer [29]. The initial energy of the neutron is $E_{0}=750 \mathrm{meV}$. The black line (parabola) is the calculated recoil trajectory of a free proton. The strong intensity contributions with $E \approx 0$ are mainly due to the aluminum sample cell used in this experiment. The intensity peak centered at $E \approx 420 \mathrm{meV}$ and $K \approx 14 \AA^{-1}$ is due to the vibrational $\mathrm{O}-\mathrm{H}$ stretching modes and is of particular interest for the investigations reported in this paper. Further details of these and other intensity peaks are discussed in the text. (Reproduced from Ref. [21] with permission.)

We now consider the intensity peak around the energy transfer $E=200 \mathrm{meV}$, which is due to the excitation of the bending vibrational mode. This mode is well known, especially from the IRspectroscopic studies. The interesting point here is that this peak does not lie on the calculated free $\mathrm{H}$-recoil parabola, but is positioned at higher $K$-values, i.e., it exhibits a positive $K$-shift. However, this result also has a simple conventional explanation: The vibrational motion of $\mathrm{H}$ in the $\mathrm{H}$-bonded system of ice should be hindered by the adjacent molecules and thus the struck $\mathrm{H}$ atom may become "dressed" by environmental degrees of freedom. Hence, it is natural to expect that its effective mass for the neutron-H collision should be increased. This is indeed the case here - an estimate derived from the peak- centroid position, presented in Figure 3, yields $M_{H}^{\text {eff }} \approx 1.3$ a.m.u. for the effective mass of the struck $\mathrm{H}$ atom. [Of course, a neutron-O collision may also excite this vibration. However, its contribution to the measured intensity is much less than that of neutron-H collisions.]

In other words, we may conclude directly that the bending vibration is not decoupled from the other dynamical modes (i.e., librations, phonons, etc.) of the complex H-bonded ice system. Clearly, although this is in contrast to the aforementioned behavior of the neutron- $\mathrm{H}$ collision exciting the stretching modes, both observations have a (qualitative) conventional theoretical interpretation [1, 23].

The preceding comparison of the two well-known vibrational modes of $\mathrm{H}_{2} \mathrm{O}$, as derived from the measured $K-E$ intensity data, demonstrates the corresponding advantage of the INS method over 
the common optical methods of IR and Raman spectroscopy, which cannot measure momentum transfers.

\subsection{INS from Oriented Single $\mathrm{H}_{2} \mathrm{O}$ Molecules in Nanocavities}

In this section, we consider the INS results obtained [31] from the recent investigations carried out using the TOF instrument SEQUOIA [32] (SNS Facility, Oak Ridge National Laboratory, USA). Figure 4 shows the measured INS intensity maps from single $\mathrm{H}_{2} \mathrm{O}$ molecules confined in nanoscopic channels of a single crystal of beryl $\left(\mathrm{Be}_{3} \mathrm{Al}_{2} \mathrm{Si}_{6} \mathrm{O}_{18}\right)$ along its $c$ axis, having a diameter of about $4.86 \AA$. The molecules are oriented with their $\mathrm{H}-\mathrm{H}$ direction parallel to the $c$-axis of the crystalline channels [31].

Two related INS experiments were performed separately, one with $K$ perpendicular, and another with $K$ parallel to the $c$-axis, in order to study the directional variations in the $\mathrm{OH}$-vibrational dynamics and the associated Born-Oppenheimer potential along the measured directions (see [31] for more details). The data shown in Figure 4 represents the difference between the two $K$ orientations with respect to the $c$-axis.

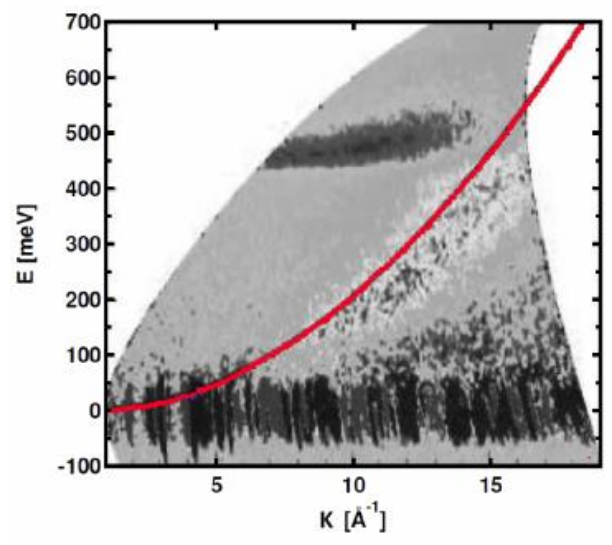

Figure 4 INS from oriented single $\mathrm{H}_{2} \mathrm{O}$ molecules in the sub-nanometer channels of beryl at $T=7 \mathrm{~K}$, recorded using the SEQUOIA spectrometer. The incident energy of the neutron was $E_{0}=800 \mathrm{meV}$. The figure shows the difference between the two measured $S(K, E)$ maps for (1) $K$-perpendicular-to- $c$ and (2) $K$-parallel-to- $c$ orientation. The red parabola line represents the calculated recoil trajectory of a free proton. The strong intensity peak centered at $420 \mathrm{meV}$ is the well-known $\mathrm{O}-\mathrm{H}$ stretching vibrational mode (Adapted from Figure 7 of [31].)

The comparison of these INS results with the preceding ones obtained in the case of bulk ice clearly demonstrates the striking effect of the momentum-transfer deficit being of central importance for this paper, which concerns the $\mathrm{O}-\mathrm{H}$ stretching vibrations of the water molecule. (cf. Figure 4).

We first consider the observed intensities in the energy-transfer range of $150-400 \mathrm{meV}$. As can be noticed from the previous discussions in the subsections above, the bending vibrational mode of $\mathrm{H}_{2} \mathrm{O}$ lies in this energy range. The INS data qualitatively exhibits the same positive $K$-shift as already observed in the case of bulk ice, which is in complete agreement with the conventional theoretical 
expectations. It should be noted that this effect is present in the INS spectra measured with both the aforementioned $K$ orientations [31].

In contrast, however, the stretching vibrational intensity shows quite the opposite $K$-shift behavior, i.e., the centroid of the peak, in this case, is shifted significantly to smaller $K$-values than expected according to the conventional theories. Here the measured intensity peak is strongly shifted to the "left" of the peak corresponding to the recoil of the free $\mathrm{H}$ atom. As shown in [31], both crystal orientations mentioned above exhibit this striking effect.

[The reader who is interested in additional experimental details may notice the following point. Due to the observed difference in the two intensity maps (see also the legend of Figure 4) and since the peak corresponding to the $K$-parallel-to- $c$ peak orientation is much stronger than that corresponding to the other orientation, the difference in the intensities shown in [31] appears to be negative, which of course is of no relevance here.]

This striking $\hbar K$-transfer deficit is large enough to be clearly seen in the measured raw data. The stretching vibrational peak at $E \approx 470 \mathrm{meV}$ is measured to be around $K \approx 10-12 \AA^{-1}$, in clear contrast to the conventional free $\mathrm{H}$-recoil value of $K_{r} \approx 15 \AA^{-1}$. The corresponding effective mass of $\mathrm{H}$ atom participating in the $\mathrm{O}-\mathrm{H}$ stretching vibration is then estimated to be

$$
M_{H}^{\text {eff }} \approx 0.55 \pm 0.1 \text { a.m.u. }
$$

This experimental result is even qualitatively in disagreement with conventional theory (see subsections 2.1 and 2.2).

\subsubsection{A Second Related Experiment}

It should be mentioned here that the above-mentioned effect has been recently confirmed by a similar experiment of water molecules in a single crystal of cordierite $\left(\mathrm{Al}_{4} \mathrm{Mg}_{2} \mathrm{Si}_{5} \mathrm{O}_{18}\right)$ [33]. This material has channels with a slightly larger diameter of about $5.50 \AA$. In addition, in this experiment, the numerical value of the observed momentum-transfer deficit obtained by the exciting the molecular stretching modes is virtually the same as in the experiment with beryl.

It should also be noted that the observed $\hbar K$-transfer deficit is much larger than any conceivable experimental error.

\subsubsection{Bending Vibration of $\mathrm{H}_{2} \mathrm{O}$ Molecules Adsorbed onto $\mathrm{Al}_{2} \mathrm{O}_{3}$ Nanoparticles}

In this context, a remarkably different behavior of the $\mathrm{HOH}$ bending vibrational mode (also called the scissors mode) of $\mathrm{H}_{2} \mathrm{O}$ molecules in hydrated $\gamma-\mathrm{Al}_{2} \mathrm{O}_{3}$ nanoparticles (also known as $\gamma$-alumina) should be stressed. This experiment was also carried out using the SEQUOIA spectrometer by Spencer and colleagues [34]. Many samples having different pore diameters (ca. 5-30 nm) have been investigated. The results allowed an unambiguous conclusion that the water content and pore size have no influence on the classical-thermodynamic behavior of this system. (The excitation energy and temperature were $E_{0}=600 \mathrm{meV}$ and $T=7 \mathrm{~K}$ respectively.)

We now concentrate on the bending vibrational mode, which here is centered at ca. $E \approx 205 \mathrm{meV}$ and $K \approx 7-10 \AA^{-1}$. In clear contrast to the related findings [31] discussed above which showed a 
conventional behavior of bending vibration, visual inspection of the figure showing the INS data [34] (not reproduced here) revealed the presence of an "anomalous" momentum-transfer deficit in the bending vibrational mode as well (precise numerical values cannot be extracted from the shown intensity maps). The roughly estimated value of the momentum transfer of $K \approx 7-10 \AA^{-1}$, is markedly smaller than that obtained in the ice-Ih measurement reported above, as well as that in the related data of $\mathrm{H}_{2} \mathrm{O}$-beryl system [31] shown in Figure 4.

\subsection{INS from Single $\mathrm{H}_{2}$ Molecules in C-Nanotubes - Roto-Translational Motion}

A related and an even stronger "anomalous" INS result has been recently observed by Olsen et al., [35] from single $\mathrm{H}_{2}$ molecules adsorbed in multi-walled nanoporous carbon (pore diameter $\approx 8-20 \AA$ ). undergoing rotational and translational motion.

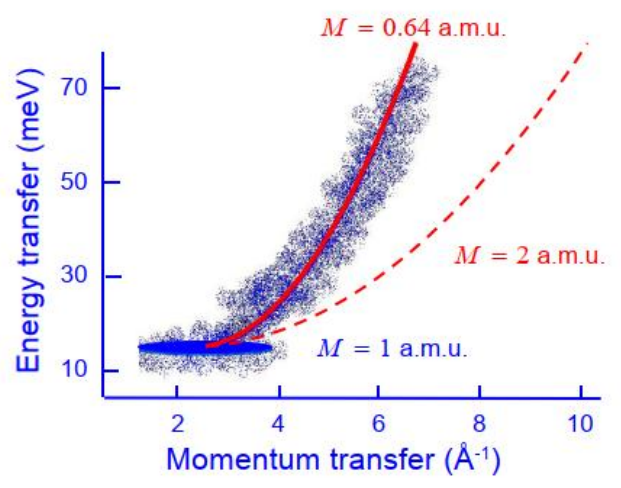

Figure 5 Schematic representation of the INS results from $\mathrm{H}_{2}$ molecules in carbon nanotubes, adapted from Figure 1 of Ref [35]. The initial energy of neutron was $E_{0}=90$ meV and its temperature was $T=23 \mathrm{~K}$. The broad intensity band is due to the translational motion of the recoiling $\mathrm{H}_{2}$ molecules, also called "roto-recoil" (white-blue ribbon). It starts at the first rotational excitation of the $\mathrm{H}_{2}$ molecule, which is centered at $E_{\text {rot }} \approx 14.7 \mathrm{meV}$ and $K_{\text {rot }} \approx 2.7 \AA^{-1}$ (blue ellipsoid). The $K-E$ position of the rotational excitation is in complete agreement with the conventional theory. In clear contrast, the detailed data analysis by the authors of [35] provides a fit (red parabola, full thick line) to the roto-recoil data, which exhibits a striking reduction of the effective mass of the recoiling $\mathrm{H}_{2}$ molecule. The data are consistent with the effective mass of only 0.64 a.m.u. of the translating $\mathrm{H}_{2}$ molecule. A comparison with the conventional expectations of $\mathrm{H}_{2}$ translation is given by the red thin (dashed) line, which represents the recoil parabola with an effective mass of 2.0 a.m.u. For full details of the data analysis and further experimental data, see [35]. (Reproduced with permission from Ref. [13].)

The INS experiments were carried out using the ARCS TOF spectrometer (SNS, Oak Ridge Nat. Lab., USA) [26]. This is also a two-dimensional spectrometer, and its detectors (about 100,000) measure a broader area of the $K-E$ plane than that measured by the previously mentioned SEQUOIA spectrometer. The experiments were carried out at $T=23 \mathrm{~K}$, with an incident neutron energy of 
$E_{0}=90 \mathrm{meV}$. Hence, the maximally possible E-transfer cannot excite (the much higher lying) molecular vibrations, but only excites rotation and translation of $\mathrm{H}_{2}$ which interacts only weakly with the substrate:

$$
E=E_{\text {rot }}+E_{\text {trans }}
$$

The experimental two-dimensional INS intensity map, $S(K, E)$, is schematically shown in Figure 5 , which has been adapted from the original paper [35]. One may notice the following features: First, the intensive peak with the energy around $E_{\text {rot }} \approx 14.7 \mathrm{meV}$ is due to the well known rotational excitation $J=0 \rightarrow 1$ of a free $\mathrm{H}_{2}$ molecule (see, e.g., [23]). The $K$-transfer of this intensity peak is $K_{\text {rot }} \approx 2.7 \AA^{-1}$. It follows that the peak position in the $K-E$ plane is completely in line with the conventionally expected numerical value of the $\mathrm{H}$-mass. In other words, the data fulfills the relation $E_{\text {rot }}=\left(\hbar K_{\text {rot }}\right)^{2} / 2 M_{H}$ with where $M_{H}$ being is the mass of the free $\mathrm{H}$ atom,

$$
\text { rotation: } M_{H}=1.0079 \text { a.m.u. }
$$

or, in other words, $M_{e f f}(H)=M_{H}$. This is also in line with the aforementioned viewpoint that in INS each neutron scatters from a single $H$ atom $[23,24]$.

A detailed analysis of the measured roto-recoil data, shown in Figure 5, revealed a significant $K$ transfer deficit and the associated strongly reduced effective mass of the whole translating $\mathrm{H}_{2}$ molecule (red parabola, full line; cf. Eq. (1)):

$$
\text { translation (recoil): } \quad M^{e f f}\left(\mathrm{H}_{2}\right) \approx 0.64 \pm 0.07 \text { a.m.u. }
$$

This observation contradicts the conventionally expected value $M\left(\mathrm{H}_{2}\right)=2.01$ a.m.u. for a freely recoiling (translating) $\mathrm{H}_{2}$ molecule (right parabola, red dashed line). An extensive numerical analysis of the data and the expected conventional results being violated by the experiment are presented in [35].

The highly reduced effective mass is far beyond any conceivable experimental error. In particular, the observed momentum-transfer deficit is about $-43 \%$ of the conventionally expected value, thus providing further experimental evidence of the existence of the new momentum-transfer deficit under consideration in this article.

As discussed in the preceding subsections, explained in more detail in [13, 14], and according to the conventional theory, every $\mathrm{H}_{2}$-substrate binding and/or interaction must correspond to an increased effective mass of the scattering system (particle). However, as we will see below, the observed decreased effective $\mathrm{H}$-mass finds a straightforward interpretation in the framework of the modern theories of WV and TSVF.

The experimental results of this and the preceding subsections demonstrate the power and/or usefulness of the new two-dimensional instruments. This experimental technique represents a powerful method, revealing novel aspects of quantum dynamics. This is owing to the fact that the $K$ and $E$ transfers can be measured over a broad range of the $K-E$ plane. This provides an advantage making these spectrometers superior to the common one-dimensional ones (e.g., TOSCA [36]), in which the detector(s) can only measure along a single specific trajectory in the $K-E$ plane. [In fact, TOSCA can measure along two such trajectories [36], but this detail is unimportant in the present context.] 
In order to demonstrate the validity of the remarks made above, one may consider the results of a similar experiment [37] of $\mathrm{H}_{2}$ molecules adsorbed in single-wall carbon nanotubes at $T \approx 20 \mathrm{~K}$, investigated with the above-mentioned one-dimensional spectrometer TOSCA. This work also reports the results of the roto-recoil INS spectrum, however, only of the $E$-dependence of the measured intensity. In the associated theoretical analysis, the mass of the $\mathrm{H}_{2}$ molecule was fixed to its conventionally expected value of 2 a.m.u. Hence the strong anomalous $K$-transfer deficit remained unnoticed. (see [37], p. 903.)

\subsection{Experimental Findings and Their Significance}

We have presented result on the new quantum effect of momentum-transfer deficit in the quantum dynamics of the neutron-H collisions. The local environment of the struck $\mathrm{H}$ atom appears to contribute to this effect. In particular, this is supported by the fact that the stretching vibrational modes of the $\mathrm{H}_{2} \mathrm{O}$ molecule in two different nano-structured materials exhibit this effect, which is absent in the case of bulk ice-lh. A qualitatively similar conclusion is obtained for the rototranslational motion of $\mathrm{H}_{2}$ molecules in C-nanotubes. These observations cannot have a conventional interpretation since the effective mass of the struck $\mathrm{H}$ atom, being initially "at rest" (or, in thermodynamic equilibrium), turns out to be considerably smaller than 1 a.m.u. (i.e., the mass of a free $\mathrm{H}$ atom), and that of the translating $\mathrm{H}_{2}$ molecule is much smaller than 2 a.m.u. (i.e., the mass of a free $\mathrm{H}_{2}$ molecule).

The theoretical investigations presented in the following sections provide a straightforward interpretation of these observations.

(I) Within the theoretical framework of WV and TSVF (see Theoretical Part I), this effect is shown to be directly connected with the weak value of the momentum operator of $\mathrm{H}$ and associated deformation of the final-state wavefunction of struck $\mathrm{H}$. In other words, the observed momentumtransfer deficit makes possible

(a) the direct experimental determination of the width of the final state in the $\mathrm{H}$ atom

(in the momentum space); see subsections 3.3 and 3.4. It should be emphasized that such a possibility does not exist with the presently known neutron scattering experimental methods.

(II) Furthermore, within the framework of the quantumness of correlations (see Theoretical Part II), the observed $\hbar K$-deficit appears to be directly linked with

(b) the negative conditional entropy and the number of quantum bits (qubits)

capturing the information-theoretic content of the H-environment interaction and which are participating and/or "consumed" in the complex quantum dynamics of INS (see subsections 4.7.5 and 4.8).

\section{Theoretical Part I: Quantum Scattering in View of Weak Values}

\subsection{Weak Values (WV), Two-State-Vector-Formalism (TSVF) and Impulsive Measurements}

Let us start with the following short remarks about the new theory of WV and TSVF. Consider the well-known equation for the expectation value of standard quantum mechanics, 


$$
\langle A\rangle=\left\langle\psi_{i}|\hat{A}| \psi_{i}\right\rangle
$$

where $\hat{A}$ is the operator (i.e., an observable) whose value is to be measured and $\left|\psi_{i}\right\rangle$ is the initial state of the system (just before the measurement). As $\hat{A}$ is hermitean, $\langle A\rangle$ is a real number.

Now consider the formal expression

$$
A^{w} \equiv \frac{\left\langle\psi_{f}|\hat{A}| \psi_{i}\right\rangle}{\left\langle\psi_{f} \mid \psi_{i}\right\rangle}
$$

where $\left|\psi_{f}\right\rangle$ is the final state of the system (i.e., just after the measurement). In general, $A^{w}$ is a complex number and is the weak value of $\hat{A}$ of a system experimentally prepared in the state $\left|\psi_{i}\right\rangle$ and experimentally post-selected in the state $\left|\psi_{f}\right\rangle$ [2]. It should be emphasized that both the real and imaginary parts of $A^{w}$ are experimentally accessible quantities (see, e.g., the review article [12]).

One immediately sees that if $\left|\psi_{i}\right\rangle=\left|\psi_{f}\right\rangle$, then $\langle A\rangle=A^{w}$. Thus, Eq. (18) represents a generalization of the standard definition (Eq. (18)).

Furthermore, consider again the conventional expectation value of an observable $\hat{A}$, Eq. (18), and suppose that $\left|\psi_{i}\right\rangle=\sum_{j} c_{j}|j\rangle$, where the kets $\{|j\rangle\}$ represent an orthogonal basis. Let us assume that we make a measurement of the observable $\hat{A}$ with respect to the above basis and make a postselection of the results for the final state being $|j\rangle$. I.e., we make a strong projective measurement described by the projector $|j\rangle\langle j|$. Usually, one is interested in the mean value, $\left\langle\psi_{i}|\hat{A}| \psi_{i}\right\rangle$, of the measuring results which can be simply reformulated as follows:

$$
\left\langle\psi_{i}|\hat{A}| \psi_{i}\right\rangle=\sum_{j} c_{j}^{*}\left\langle j|\hat{A}| \psi_{i}\right\rangle=\sum_{j}\left|c_{j}\right|^{2} \frac{\left\langle j|\hat{A}| \psi_{i}\right\rangle}{\left\langle j \mid \psi_{i}\right\rangle} \equiv \sum_{j}\left|c_{j}\right|^{2} A_{j, \psi_{i}}^{w}
$$

Here, $A_{j, \psi_{i}}^{w}$ is the WV of the observable $\hat{A}$ with respect to the pre-selected state $\left|\psi_{i}\right\rangle$ and the postselected state $|j\rangle .\left|c_{j}\right|^{2}$ is the probability for the occurrence of $|j\rangle$; cf. [6]. These equalities show that the specific effects which may be captured by the weak values $A_{j, \psi_{i}}^{w}$ may become "smeared out" in the common expectation value $\left\langle\psi_{i}|\hat{A}| \psi_{i}\right\rangle$ of conventional quantum mechanics, presuming that the individual weak values do possess some physical meaning (which is indeed the case).

Indeed, Aharonov and collaborators have revealed the crucial physical significance of Eq. (19). I.e., in specific and well defined experimental setups, weak values are new measurable quantities [2]! This corresponds to a novelty, or discovery, since weak values are unknown in standard quantum mechanics (cf. e.g., the Feynman Lectures [22]).

The measurement in the WV-TSVF theory is described by an impulsive von Neumann process [38], introducing a pointer (or measuring apparatus) with a wavefunction $|\Phi\rangle$. It should be emphasized that a pointer is a quantum object as well. Let the interaction Hamiltonian be

$$
\widehat{H}_{i n t}=g \delta\left(t-t_{0}\right) \hat{A} \otimes \widehat{M}
$$

where $g$ is a (small and real) coupling constant, $\delta\left(t-t_{0}\right)$ is the delta function and $\widehat{M}$ is the operator of the coupling quantity of the pointer. $\hat{A}$ is the operator of the system's quantity that is to be measured. The interaction is impulsive, and thus one is interested in the time evolution during a short time interval around $t_{0}$, which allows neglecting (in the calculations) the "free" evolution before and after $t_{0}$. 
The standard von Neumann (called strong) measurements [38] yield the eigenvalues of the measured observable and at the same time (strongly) disturb the measured system. This changes the initial state of the system and the final state is an eigenstate of the operator representing the observable. By coupling a measuring device to the observed system sufficiently weakly, i.e., by performing a weak measurement (WM), it may be possible to gain certain information from the system while limiting the disturbance induced by the measurement. Based on this idea, together with post- selection on a particular outcome of the measurement, Aharonov and collaborators [3, 4], obtained new physical insights and further experimental information from the measuring process.

Before the interaction occurs, let the initial system-apparatus state be $\psi_{i} \otimes \Phi_{i}$. After the interaction, this state evolves as

$$
\psi_{i} \otimes \Phi_{i} \rightarrow e^{-i g \hat{A} \otimes \widehat{M}} \psi_{i} \otimes \Phi_{i}
$$

( $\hbar=1$ has been temporarily put in order to shorten the notations). A crucial element of TSVF is the following: With a conventional strong measurement, we may post-select a particular final state, $\psi_{f}$, of the system. In addition, we ask for the associated final state, $\Phi_{f}$, of the measuring device (apparatus). $\Phi_{f}$ is obtained, as usual, by tracing out the dynamical variables of the system, i.e.,

$$
\Phi_{f}=\left\langle\psi_{f}\left|e^{-i g \hat{A} \otimes \widehat{M}}\right| \psi_{i}\right\rangle \Phi_{i}
$$

and for a sufficiently weak interaction (i.e., small $g$ ) one obtains by a power expansion

$$
\Phi_{f} \approx\left\langle\psi_{f}|1-i g \hat{A} \otimes \widehat{M}| \psi_{i}\right\rangle \Phi_{i}=\left\langle\psi_{f} \mid \psi_{i}\right\rangle\left(1-i g A^{w} \widehat{M}\right) \Phi_{i} \approx\left\langle\psi_{f} \mid \psi_{i}\right\rangle e^{-i g A^{w} \widehat{M}} \Phi_{i}
$$

Here, $A^{w}$ is the aforementioned WV of the observable $\hat{A}$. Furthermore, one assumes $\left\langle\psi_{f} \mid \psi_{i}\right\rangle \neq 0$. Hence, the state of the measuring device appears to evolve with the aid of an effective one-body Hamiltonian $\widehat{H}_{M}=g A^{w} \widehat{M}$, i.e.,

$$
\Phi_{i} \rightarrow e^{-i g A^{w} \widehat{M}} \Phi_{i} .
$$

Thus, we arrive at the interesting result that the pointer dynamics depends on the three dynamical quantities $\hat{A},\left|\psi_{i}\right\rangle$, and $\left|\psi_{f}\right\rangle$ only through the single complex number $A^{w}$.

In order to apply the theory to the neutron-atom scattering process [13], in the following theoretical derivations, we will make the choice

$$
\widehat{M}=\hat{q}
$$

i.e., the pointer variable will be its position. Furthermore, for the atomic operator quantity, we will use

$$
\hat{A}=\hat{P},
$$

i.e., the momentum operator of the struck atom (or nucleus). According to the general WV-TSVF theory, the observed mean pointer momentum after the interaction changes from its initial numerical value, $\langle p\rangle_{i}$, to its final value, and this change is expressed as

$$
\langle p\rangle_{f}=\langle p\rangle_{i}+g \operatorname{Re}\left[P^{w}\right]
$$


which contains the real part of the WV of the atomic momentum $P$. For the full derivations, see e.g., $[6,39]$.

\subsubsection{An Additional Result Concerning the Meaning of WV and TSVF}

To shed more light upon the physical interpretation and/or the formal structure of the new WVTSVF theory, the following observation may be helpful. Here, we start from the von Neumann-type impulsive interaction Hamiltonian, Eq. (21), and arrive at an insightful result derived by Pati and Wu [40].

First, we will make use of the following formula (see, e.g., [40] or [2], p. 39)

$$
\hat{A}\left|\psi_{i}\right\rangle=\langle\hat{A}\rangle\left|\psi_{i}\right\rangle+\Delta \hat{A}\left|\bar{\psi}_{i}\right\rangle
$$

where $\left|\bar{\psi}_{i}\right\rangle$ is a state orthogonal to $\left|\psi_{i}\right\rangle,\langle\hat{A}\rangle=\left\langle\psi_{i}|\hat{A}| \psi_{i}\right\rangle$, and $\Delta \hat{A}$ is the quantum uncertainty in the state $\left|\psi_{i}\right\rangle$, i.e., $(\Delta \hat{A})^{2}=\left\langle\psi_{i}\left|(\hat{A}-\langle\hat{A}\rangle)^{2}\right| \psi_{i}\right\rangle$. Operating $\left\langle\psi_{f}\right|$ on both sides of Eq. (29), we get

$$
A^{w}=\langle\hat{A}\rangle+\Delta \hat{A} \frac{\left\langle\psi_{f} \mid \bar{\psi}_{i}\right\rangle}{\left\langle\psi_{f} \mid \psi_{i}\right\rangle} \equiv\langle\hat{A}\rangle+\delta A_{w}
$$

This result additionally sheds light on a crucial reason for the non-vanishing difference, $\delta A_{w}$, between the conventional expectation value $\langle\hat{A}\rangle$ and the newly introduced $\mathrm{WV} A^{w}$. The reason is given by a non-vanishing uncertainty $\Delta \hat{A}>0$ in connection with a non-vanishing scalar product $\left\langle\psi_{f} \mid \bar{\psi}_{i}\right\rangle \neq 0$. The physical interpretation of the latter is that the two states $\left|\psi_{f}\right\rangle$ and $\left|\bar{\psi}_{i}\right\rangle$ possess the ability to produce quantum interferences.

To summarize, this concise derivation demonstrates that the novel features of WV and TSVF are intrinsically related to the quantum interference between the post-selected state and another quantum state that is orthogonal to the pre-selected state [40]. In other words, this result also stresses the importance of both concepts: the conventional "pre-selected initial state" and the "postselected final state".

\subsection{A Didactical Example: Photon-Mirror Anomalous Momentum Transfer in an Interferometer}

Here, we consider a surprisingly simple theoretical model by Aharonov et al. [41], in which the concept of weak values emerges in a natural way and which also motivated many of our investigations. Here, we follow the presentation in Refs. [14, 15].

A specific result of [41] concerns the "anomalous" momentum transfer between two quantum objects: a photon and a reflecting mirror. Hence, it appears to be in intimate connection with our observation of the momentum- transfer deficit observed in the neutron- $\mathrm{H}$ collisions presented in the Experimental Part (Section 2). The schematic representation of the experimental setup is shown in Figure 6.

A beam of photons (or particles) enters a device similar to a usual Mach-Zehnder interferometer (MZI). However, one is interested in the case where one reflecting mirror, $M$, is sufficiently small and represents a quantum object. Its momentum distribution is assumed to be experimentally accessible in an appropriate non-demolition measurement [42]. In Ref. [41], the authors derived the following 
astonishing result: Although it is clear that the photons collide with the mirror only from inside the MZI, they do not push it outwards, but instead succeed in pulling it in. It is obvious that this result cannot have any conventional theoretical interpretation (neither classical nor quantum).

Here, we outline the theoretical derivation of this effect, following closely the presentation of Ref. [41]. Two beam splitters have the same reflectivity, $r$, and transmissivity, $t$, (assumed real, and assuming that the condition $r^{2}+t^{2}=1$ holds). Here, we assume that $r>t$. Hence, when a single photon (particle) enters the $\mathrm{MZI}$ by impinging on the left side of the beam splitter, $\mathrm{BS}_{1}$, as shown in Figure 6 , the (classical) probabilities to take the path $A$ or $B$ are $r^{2}$ and $t^{2}$, respectively.

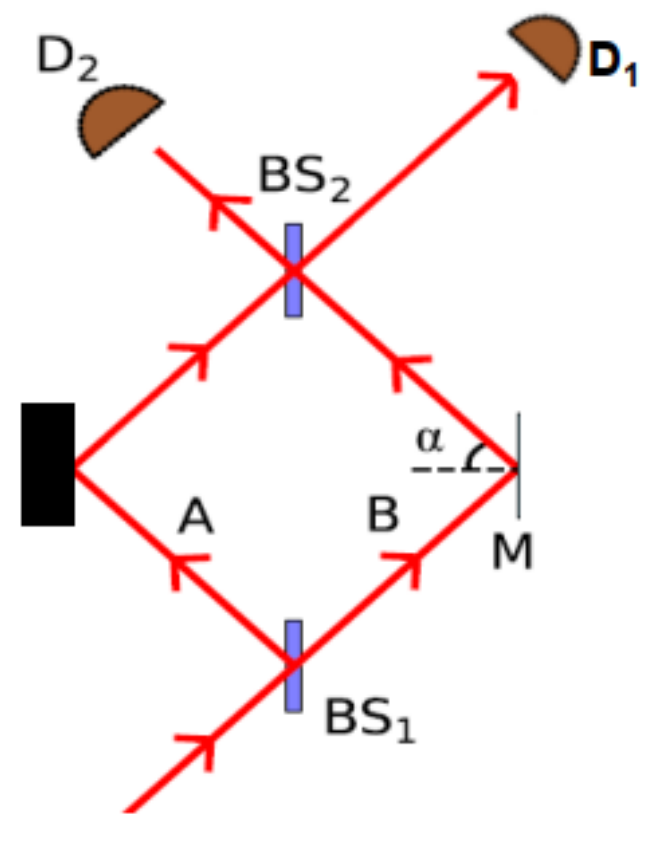

Figure 6 Path of a light beam (a photon, a particle, etc.) in a Mach-Zehnder interferometer (MZI). The mirror, $\mathrm{M}$, is a microscopic quantum object. The theoretical analysis concerns the photons (particles) emerging toward detector $D_{2}$. (Reproduced from Ref. [14] with permission.)

According to standard quantum mechanics, an incoming state $\mid$ in $\rangle$ impinging on a beam splitter will emerge as a superposition of a reflected state $|R\rangle$ and a transmitted state $|T\rangle$, i.e., $|i n\rangle \rightarrow \operatorname{ir}|R\rangle+$ $t|T\rangle$. Thus, when a photon impinges from the left on $\mathrm{BS}_{1}$, (see Figure 6), the effect of the beam splitter is to produce a linear superposition of photon states inside the interferometer

$$
|\Psi\rangle=\operatorname{ir}|A\rangle+t|B\rangle
$$

$|A\rangle$ and $|B\rangle$ denote the photon propagating along the arms $A$ and $B$ arms respectively of the MZI .

As assumed above, the second beam splitter, $\mathrm{BS}_{2}$, should be identical to $\mathrm{BS}_{1}$. It can be easily seen that a photon in the state $\left|\Phi_{1}\right\rangle=t|A\rangle-i r|B\rangle$ impinging on $\mathrm{BS}_{2}$ will emerge toward the detector $\mathrm{D}_{1}$. Correspondingly, a photon in the orthogonal state $\left|\Phi_{2}\right\rangle=-i r|A\rangle+t|B\rangle$ will emerge toward detector $\mathrm{D}_{2}$. 
In the case of a classical photon (or beam), it is obvious that this pushes the mirror $M$ outward. In contrast, in the case of a quantum beam of photons (or particles) a strange effect may happen. Each photon incident on $M$ gives it a momentum kick $\delta$. [It may be noted that each momentum kick must be much smaller than the spread $\Delta$ of momentum of the mirror. This is a general property of any interferometric experiment, in order to maintain the coherence of the beam in the MZI.]

Let $\phi(p)$ be the initial quantum state of $M$ and $|\Psi\rangle$ the quantum state of the photon after the input beam splitter, $\mathrm{BS}_{1}$, but before reaching $M$. The reflection from the mirror, results in the quantum state:

$$
|\Psi\rangle \phi(p)=(\operatorname{ir}|A\rangle+t|B\rangle) \phi(p) \rightarrow \operatorname{ir}|A\rangle \phi(p)+t|B\rangle \phi(p-\delta)
$$

Of course, this holds before the photon reaches the second beam splitter $\mathrm{BS}_{2}$.

If the kick, $\delta$, is strong enough, $\phi(p)$ is orthogonal to $\phi(p-\delta)$, and the photon ends up entangled with the mirror, which implies that coherence is lost.

As an example, we assume the state of the mirror to be (up to normalization) $\phi(p)=\exp \left(-\frac{p^{2}}{2 \Delta^{2}}\right)$. We consider now a single photon propagating through the interferometer, assuming that the photon's kick is small, i.e., $\delta \ll \Delta$. Then, we can approximate the combined state (32) of the photon and the mirror just before the photon reaches the output beam splitter by

$$
|\Psi\rangle \phi(p) \approx \operatorname{ir}|A\rangle \phi(p)+t|B\rangle\left(\phi(p)-\frac{d \phi(p)}{d p} \delta\right)=|\Psi\rangle \phi(p)-t|B\rangle \frac{d \phi(p)}{d p} \delta
$$

We now consider the following particular case which may appear to be "innocent" or "without any particular physical interest": We analyze the effect caused by photons emerging in the beam directed toward $\mathrm{D}_{2}$ only. (Clearly, modern single-photon counting setups can do this.)

The state of the mirror, $M$, is then obtained by projecting the joint state onto the state of the photon corresponding to this beam, i.e., (up to normalization)

$$
\begin{aligned}
\left\langle\Phi _ { 2 } \left|\left(|\Psi\rangle \phi(p)-t|B\rangle \frac{d \phi(p)}{d p} \delta\right)\right.\right. & =\left\langle\Phi_{2} \mid \Psi\right\rangle\left(\phi(p)-\frac{t\left\langle\Phi_{2} \mid B\right\rangle}{\left\langle\Phi_{2} \mid \Psi\right\rangle} \frac{d \phi(p)}{d p} \delta\right) \\
& =\left\langle\Phi_{2} \mid \Psi\right\rangle\left(\phi(p) \frac{\left\langle\Phi_{2}\left|\mathrm{P}_{B}\right| \Psi\right\rangle}{\left\langle\Phi_{2} \mid \Psi\right\rangle} \frac{d \phi(p)}{d p} \delta\right) \\
& =\left\langle\Phi_{2} \mid \Psi\right\rangle \phi\left(p-P_{B}^{W} \delta\right)
\end{aligned}
$$

Here $\mathbf{P}_{B}=|B\rangle\langle B|$ is the projection operator on the state $|B\rangle$ and

$$
P_{B}^{W}=\frac{\left\langle\Phi_{2}\left|\mathbf{P}_{B}\right| \Psi\right\rangle}{\left\langle\Phi_{2} \mid \Psi\right\rangle}
$$

is the weak value (WV) of $\mathbf{P}_{B}$ with respect to the initial $|\Psi\rangle$ and the final $\left|\Phi_{2}\right\rangle$ states $[2,4-7,12]$.

The numerical value of $P_{B}^{W}$ is calculated in a straightforward manner:

$$
\begin{aligned}
P_{B}^{W}=\frac{\left\langle\Phi_{2}\left|P_{B}\right| \Psi\right\rangle}{\left\langle\Phi_{2} \mid \Psi\right\rangle} & =\frac{\left(i r \left\langleA|+t\langle B|) P_{B}(i r|A\rangle+t|B\rangle)\right.\right.}{(i r\langle A|+t\langle B|)(i r|A\rangle+t|B\rangle)} \\
& =-\frac{t^{2}}{r^{2}-t^{2}}
\end{aligned}
$$

Therefore, the momentum kick received by the mirror due to a photon emerging toward $D_{2}$ is

$$
\delta p_{M}=P_{B}^{w} \delta=-\frac{t^{2}}{r^{2}-t^{2}} \delta
$$


We have assumed above that $r>t$, and therefore, the sign of the momentum received by the mirror is here negative. Hence, we arrive at the striking result that the mirror is pushed toward the inside of the MZI. This surprising momentum change is the result of the mirror receiving the following superposition: a kick $\delta$ and no kick at all, corresponding to the photon (particle) propagating through the two MZI arms. For further details, see [41].

To summarize, the photons that exit the $M Z I$ and end up in the detector $D_{2}$ give negative momentum kicks to the mirror $M$, although they collide with the mirror only from the inside of the MZI. Hence, these photons do not push the mirror outwards, but rather somehow succeed to pull it in. As Aharonov et al., discussed this result, this is a purely quantum effect being realized by a quantum superposition of giving the mirror zero momentum and positive momentum - the superposition causes the striking effect that the mirror gains a negative momentum [41].

\subsection{Incoherent Scattering in the Theoretical Framework of WV and TSVF}

In this subsection, we follow the main theoretical treatment presented in the original paper [13].

\subsubsection{Neutron-Atom Collision, Model Hamiltonian}

First, we outline the main result derived from the WV-TSVF theory in the context of non-relativistic incoherent scattering, particularly in the physical context of INS, as originally explored in Ref. [13] (see also [15]).

Let $(q, p)$ be the dynamical variables of the position and momentum of the probe particle (here a neutron; this corresponds to the pointer in the general theory). Similarly, let $(Q, P)$ are be the position and momentum of the investigated system (e.g., an atom). We also use the notation that $\hat{Y}$ represents the operator of the physical observable $Y$.

To simplify the notations in the derivations, it suffices to consider the one-dimensional dynamics along the direction of the momentum transfer $\hbar \mathrm{K}$. Then, $\hat{P}$ denotes the operator of the momentum component along $\mathbf{K}$ of the (nucleus) atom.

Momentum conservation in the two-body neutron-atom collision implies (cf. Eq. (7))

$$
-\hbar K_{n}=+\hbar K_{A} \equiv \hbar K>0
$$

where $+\hbar K_{A} \equiv \hbar K$ is the momentum transfer to the atom and $-\hbar K_{n}$ is the associated momentum transfer to the neutron. Following the standard convention (cf. [1]), $K_{A}$ is chosen to be positive.

In [13] we discussed and heuristically derived a von Neumann-type interaction Hamiltonian describing the momentum and energy transfers due to the collision.

However, motivated by the WV-TSVF theory, one may expect "small" deviations from the conventionally expected $K$ - and $E$-transfers. As discussed in $[13,15]$, these deviations can be formally captured by introducing a small momentum difference, $\hat{P}-\hbar K \hat{I}_{A}$, into the interaction Hamiltonian, where $\hat{I}_{A}$ : is the identity operator in the atomic subspace. Finally, the von Neumann-type model interaction Hamiltonian that captures the deviation from the conventional formalism is given by [13, 14]

$$
\Delta \widehat{H}_{\text {int }}(t)=+g \delta(t) \hat{q} \otimes\left(\widehat{P}-\hbar K \hat{I}_{A}\right)
$$


This model Hamiltonian has the usual (von Neumann-type) form (21) of an interaction Hamiltonian [13-15]. Assuming on physical grounds that $\hat{P}-\hbar K$ yields a suitably small contribution, in the following derivations, we formally put $g=1$.

The physical idea of introducing the aforementioned difference $\left(\hat{P}-\hbar K \hat{I}_{A}\right)$ in the interaction Hamiltonian (39) was provided by the following analogous example of Aharonov et al.: "Consider, for example, an ensemble of electrons hitting a nucleus in a particle collider. [...] The main interaction is purely electromagnetic. However, there is also a relativistic and spin-orbit correction in higher-order terms which can be manifested in the form of weak interaction." (See Ref. [43], p. 3.)

The presence of the "conventional" term, $-\hbar K \hat{I}_{A}$, in Eq. (39) causes a slight extension of the general theoretical result (28) for the total numerical values of the measured quantities, e.g.,

$$
\left[\langle\hat{p}\rangle_{f}-\langle\hat{p}\rangle_{i}\right]_{\text {total }}=\left[\langle\hat{p}\rangle_{f}-\langle\hat{p}\rangle_{i}\right]_{\text {conventional }}+\left[\langle\hat{p}\rangle_{f}-\langle\hat{p}\rangle_{i}\right]_{\text {correction }}
$$

cf. $[13,15]$.

In the following derivations, the "system" of the general formalism is given by the scattering atom and $X_{w}$ denotes a WV of the observable $\hat{X}$. Obviously, for the identity operator, $\left(\hat{I}_{A}\right)_{w}=1$ holds and thus the expression $\hat{P}-\hbar K \hat{I}_{A}$ in the above interaction Hamiltonian can be written as

$$
\left(\hat{P}-\hbar K \hat{I}_{A}\right)_{w}=P_{w}-\hbar K
$$

In order to apply the general result (Eq. (28)) to the present context, we simply need to calculate the $\mathrm{WV} P_{w}$ of $\hat{P}$ for the appropriate initial and final atomic states. Recall that the neutron here represents the "pointer" with the conjugated dynamical variables $\hat{p}$ and $\hat{q}-$ the latter occurring explicitly in the interaction Hamiltonian.

In the present physical context, it is natural to work in the momentum representation, since the momenta (and not the positions in real space) of the scatterers are usually investigated in the scattering experiments.

The scattering atom in our study (see Experimental Part) is initially in some spatially confined state. In many cases, the initial atomic wavefunction $\Xi(P)_{i}$ can be sufficiently approximated by a Gaussian, $G_{A}(P)$,

$$
\Xi(P)_{i} \approx G_{A}(P) \text {. }
$$

being centered at zero momentum, since the atom is at "rest". At low temperatures, the atom should be in its ground state and the width of $\Xi(P)_{i}$ is given by quantum uncertainty. Thus, the atom will receive a kick (described by $\hat{P}$ ) in the direction of the momentum transfer, i.e., $\hbar K_{A}=\hbar K$.

\subsubsection{Unchanged Shape of the Final Atomic State - "Anomalous" Results}

Let us now make the following reasonable assumption about the final state:

Case 1: The final state has the same width as the initial state (with both states in $P$-space). It may be noted that this assumption is widely used in the theory of molecular spectroscopy and the related quantum chemical calculations. It is physically motivated by the consideration that the collision causes a very fast transition (in the time scale of atomic or molecular motions) and, therefore, the adjacent atoms have no time to adjust to the "sudden" disturbance caused by the neutron-atom 
collision. As a matter of fact, this is also the general physical picture of the sudden approximation of standard (non-relativistic) quantum mechanics; see, e.g. [44], chapter XVII.

Hence, the final atomic state is presently assumed to have the same shape like the initial state, with the difference that now is centered at the received momentum:

$$
\Xi(P)_{f}=\Xi\left(P-\hbar K_{A}\right)_{i} .
$$

The WV of operator $\hat{P}$ is then calculated as follows:

$$
\begin{aligned}
P_{w} & =\frac{\left\langle\Xi_{f}|\hat{P}| \Xi_{i}\right\rangle}{\left\langle\Xi_{f} \mid \Xi_{i}\right\rangle}=\frac{\int d P \Xi\left(P-\hbar K_{A}\right)_{i} P E(P)_{i}}{\int d P \Xi\left(P-\hbar K_{A}\right)_{i} \Xi(P)_{i}} \\
& =+\frac{\hbar K_{A}}{2}=+\frac{\hbar K}{2}
\end{aligned}
$$

The integral in the numerator is calculated in a straightforward manner considering the following two points: $(i)$ the two $\Xi$ functions are positioned symmetrically around the average (or centroid) value, $\bar{P}=\hbar K_{A} / 2$, as one $\Xi$-function is centered at $P=0$ and the other at $P=\hbar K_{A}$; and (ii) $P$ is a linear factor in the integral. Interestingly, this result is independent of the width of $\Xi$.

This result is quite surprising, and it corresponds to a considerable momentum-transfer correction of $50 \%$. In other words, the analysis of the data of the scattered neutron will be consistent with the momentum kick given to the atom which is just half of the conventionally expected value $\hbar K_{A}$ ! That is,

$$
\left(\hat{P}-\hbar K \hat{I}_{A}\right)_{w}=+\frac{\hbar K}{2}-\hbar K=-\frac{\hbar K}{2}
$$

Based on the important general result given by Eq. (28) of the WV-TSVF $[2,4,6,12]$ theory, we thus obtain the correction

$$
\langle\hat{p}\rangle_{f}-\langle\hat{p}\rangle_{i}=\left(\hat{P}-\hbar K \hat{I}_{A}\right)_{w}=-\frac{\hbar K}{2}
$$

to the conventionally expected result. Therefore, for the measured total momentum transfer, as provided by the pointer (neutron), we obtain the result

$$
\begin{aligned}
{\left[\langle\hat{p}\rangle_{f}-\langle\hat{p}\rangle_{i}\right]_{\text {total }} } & =\left[\langle\hat{p}\rangle_{f}-\langle\hat{p}\rangle_{i}\right]_{\text {conventional }}+\left[\langle\hat{p}\rangle_{f}-\langle\hat{p}\rangle_{i}\right]_{\text {correction }} \\
& =-\hbar K+\frac{\hbar K_{A}}{2} \\
& =-\frac{\hbar K}{2}
\end{aligned}
$$

(Recall the definition $\hbar K_{A}=\hbar K$, (Eq. (8)). This expression is tantamount to the predicted effect of momentum-transfer deficit in that the absolute value of the momentum transfer on the neutron, as predicted by the preceding derivation, is smaller than the conventional theoretical prediction.

Moreover, as derived earlier [13], an assumed change in the width of the final state of an atom leads to a different amount of the predicted momentum-transfer deficit.

\subsubsection{Plane- Wave Approximation of Final Atomic State - Conventional Results}

The well-known prediction of the conventional theory is obtained by the following derivation. This also illustrates the cause of the "anomalous" momentum transfer. Here we assume, as is usually done in the conventional neutron scattering theory (see, e.g., $[1,27])$, that 
Case 2: the final state of the atom is a plane wave, i.e., it has a vanishing width in the momentum space. [Note that this is always done in the so-called impulse approximation (IA) [27].]

Remarkably, the result of the conventional theory follows here in a straightforward manner within the WV-TSVF theory, or equivalently, the possible correction due to WV-TSVF theory vanishes identically.

To prove this, we note that the final-state wavefunction of the atom in $P$-space is a delta function, $\delta_{A}$, which is centered at the assumed value of $\hbar K_{A}$, i.e.,

$$
\Xi(P)_{f}=\delta_{A}\left(P-\hbar K_{A}\right) \text {. }
$$

We then obtain for the WV of the atomic momentum, $\hat{P}$ :

$$
\begin{aligned}
P_{w} & =\frac{\left\langle\Xi_{f}|\hat{P}| \Xi_{i}\right\rangle}{\left\langle\Xi_{f} \mid \Xi_{i}\right\rangle}=\frac{\int d P \delta_{A}\left(P-\hbar K_{A}\right) P \Xi(P)_{i}}{\int d P \delta_{A}\left(P-\hbar K_{A}\right) \Xi(P)_{i}}=\frac{\hbar K_{A} \Xi\left(\hbar K_{A}\right)_{i}}{\Xi\left(\hbar K_{A}\right)_{i}} \\
& =+\hbar K_{A} \equiv+\hbar K
\end{aligned}
$$

Thus, the WV of the system's coupling operator $\left(\hat{P}-\hbar K \hat{I}_{A}\right)$ turns out to be zero:

$$
\left(\hat{P}-\hbar K \hat{I}_{A}\right)_{w}=P_{w}-\hbar K=0
$$

[Note that any additional phase factor in the final wavefunction, say $e^{\imath \chi}$, caused by the scattering, does not affect the preceding result simply because this factor cancels out in the fraction which defines the WV.]

This result implies that under the commonly used plane-wave approximation, the WV-TSVF theory yields results that are identical to those of the conventional theory. In other words,

$$
\left[\langle\hat{p}\rangle_{f}-\langle\hat{p}\rangle_{i}\right]_{\text {total }}=\left[\langle\hat{p}\rangle_{f}-\langle\hat{p}\rangle_{i}\right]_{\text {conventional }}+0=-\hbar K
$$

In simple terms, the pointer of the measuring device, which measures the momentum loss of a neutron, will exhibit the conventionally expected value of $-\hbar K$, and thus, the standard expectation of conventional theory is reproduced. This is a very satisfactory result.

Comparison of the preceding two derivations reveals the following interesting physical feature: The amount of $\hbar K$-deficit depends on the "deformation" (or change of shape) of the initial atomic state, in particular, on the width of the final state. When the final state in the momentum-space is approximately a delta function (i.e., an atomic plane wave, as is usually assumed in the conventional theory), the $\hbar K$-transfer deficit will be tiny or even unmeasurable.

This straightforward conclusion strongly affects the neutron scattering studies at higher neutron energies and the associated elevated momentum transfers, e.g., neutron Compton scattering (NCS) or deep-inelastic neutron scattering (DINS), because here the plane-wave approximation is considered (or believed) to become exactly valid; see e.g., [27, 28].

With respect to the INS experiments of the Experimental Part and their relation to the WV-TSVF theory, it should be emphasized that the post-selection of an atomic final state is done by the specific environment $\mathcal{E}$ associated with that state; see also points $(A)$ and $(B)$ of the next subsection.

\subsection{Additional Remarks I}


The novel WV-TSVF theory reveals many counterintuitive effects which may appear "strange" or even "unphysical". The explanations and remarks of this subsection may facilitate further understanding.

$(A)$ In view of the conventional theory, the new effect of the momentum-transfer deficit, as well as the apparent effective mass reduction of the scatterer, appear to violate the basic energy and momentum conservation laws. However, this statement is incorrect due to the plain fact that the scattering $\mathrm{H}$ atom represents an open quantum system, and thus the above-mentioned conservation laws do not hold strictly. Furthermore, the "environment" $\mathcal{E}$ of the struck $\mathrm{H}$ atom is also a quantum system participating actively in the neutron-H scattering process. The latter is generally true whenever the characteristic time-scale of the scattering process cannot be assumed to be identically zero. Hence, for the purpose of illustration, the "conservation relations" may be reformulated as:

$$
E_{H+\mathcal{E}}=-E_{n} \text { and } \hbar K_{H+\mathcal{E}}=-\hbar K_{n}
$$

$(B)$ The findings described in subsection 2.6, corresponding to the INS from $\mathrm{H}_{2}$ molecules in Cnanotubes, might appear to reveal a contradiction. Within the framework of the conventional theory, we obtain the following two results: $(a)$ The $J=0 \rightarrow 1$ rotational excitation of the $\mathrm{H}_{2}$ molecule exhibits the conventional $M_{\text {eff }} \approx 1$ a.m.u., as the scattering is incoherent and thus each scattered neutron exchanges energy and momentum with only one struck $\mathrm{H}$ atom . (b) On the other hand, the $M_{e f f}$ of the translational dynamics of the $\mathrm{H}_{2}$ molecule is not 2 a.m.u., - as expected by the conventional theory, due to the fact that $\mathrm{H}_{2}$ undergoes a translation "as a whole"- but $M_{e f f} \approx 0.64$ a.m.u. However, this "contradiction" fades away in light of the WV-TSVF theory, mainly due to the quantum character of the environment pointed out in $(A)$.

In other words, the above-mentioned points $(a)$ and $(b)$ simply reveal that the quantum properties of the environment of the $\mathrm{H}$ atom must be different in these two cases. This is also intuitively obvious, i.e., the locally rotating $\mathrm{H}_{2}$ is affected less by its local environment than the translating $\mathrm{H}_{2}$ molecule, which necessarily interacts with a larger piece of the $\mathrm{C}$-nanotube during its translation. Hence, the two findings $(a)$ and $(b)$ indicate that different environments effectuate different post-selections of the final state of the $\mathrm{H}_{2}$ molecule, which is almost a self-evident conclusion, if one accepts that the scattering geometry (including the sample orientation) itself "chooses" (or singles out or selects) the relevant specific features of the environment of the $\mathrm{H}_{2}$ molecule and not the theoretician who models it according to his/her beliefs.

(C) The operators $\hat{q}$ and $\hat{P}$ occurring in the Hamiltonian (Eq. (39)) are dynamical variables of two different quantum systems. This is of particular interest in view of the following important point made by Vaidman [45]: WVs arise here due to the quantum interference of an entangled wave, and these consequently do not have an analog in classical wave interference. This remark further supports the statement that a WV represents a basic quantum concept, rather than some artificially introduced quantity and/or approximation.

In clear contrast, in the conventional neutron scattering theory $[1,23,27]$, the neutron is treated as a classical object, i.e., no dynamical variable of a neutron occurs in the main theoretical formulae. For instance, consider the expression for the partial differential cross section 


$$
\frac{d^{2} \sigma_{A}}{d \Omega d E}=\mathrm{const} \cdot \frac{k_{1}}{k_{0}} b_{A}^{2} S(\mathrm{~K}, E)
$$

[where $[S(\mathrm{~K}, E)$ is the incoherent dynamic structure factor of the scatterer, (cf. Section 3), const is a constant factor, $b_{A}$ is the scattering length of nucleus $A$, and $k_{0}, k_{1}$ are defined in Eq. (3)] as well as the formulae of the various correlation functions of the standard van Hove formalism [46] (see also [1], Chapter 4). All these formulae contain the dynamical variables of only the scatterer and not of the neutron at all. That is to say that only the $c$-numbers corresponding to the neutron (i.e., $b_{A}, k_{1}$ and $k_{0}$ ) occur in the above formula. This corresponds to a decoupling of the dynamic variables of the system and the neutron, which is a feature of the first-order perturbation theory and/or the first Born approximation, which provides the theoretical framework of the conventional theory $[1,27,46]$.

[Parenthetically, it may be noted that also other scattering topics, such as X-ray inelastic scattering and electron scattering, suffer from the same weakness.]

$(D)$ In some theoretical papers, the criticism that post-selection is equivalent to "arbitrarily throwing out some data" can be found (see, e.g., [47]). However, in our experimental context of INS, a specific post-selection is tantamount to "performing a specific measurement with a well-defined resolution, using a well-specified detection setup and analyzing the measured data only". Moreover, the many applications and new predicted effects of the WV-TSVF theory clearly demonstrate that "post-selection" is a genuine physical concept, as has been stressed e.g., by Romito et al. [48].

$(E)$ Entanglement [49] and quantum interference effects are recognized to be of basic importance for quantum computing, and generally, for quantum information theory and technology (cf. [16]) and the very recent works of Refs. $[50,51])$. It would also be interesting to investigate the applicability of the new WV-TSVF theory to the field of quantum computational complexity [52, 53], which may contribute to advances in information technology (say, an optimized architecture of quantum computer chips).

In this context, WV and TSVF appear to further make available a new kind of quantum information, such as the correlation between the momentum transferred to the mirror, $M$, and the photon measured with the detector $D_{2}$ in the example of Figure 6 , the existence of which was unknown thus far. However, it must be emphasized here that the so-called "quantum supremacy" [50] of quantum computation for a fundamental problem, e.g. a nondeterministic-polynomial-complete (NP-complete) one, has not been theoretically proven until now [52-54].

$(F)$ On the Elitzur-Vaidman effect, quantum computers and the interpretation of the state vector.

As already mentioned in the introduction, "interpretational issues" are not the focus of the present article. Nevertheless, we believe that a brief discussion on these may add further physical insight into and increase the understanding of the WV-TSVF theory and the aforementioned experimental results.

An open question concerning the interpretation of quantum mechanics, say, the "true meaning" of the wavefunction, appears to be related to the experimental developments reported above.

The possibility that the wavefunction (or more generally, the state vector) is purely epistemological, i.e., solely a mathematical quantity representing the observer's knowledge, is often associated with the well-known Copenhagen interpretation, sometimes also called the "orthodox" interpretation of quantum mechanics. An ingredient of this is to consider the state vector to be associated with an ensemble of quantum systems, not with the individual ones. 
The alternative, and recently emerging, viewpoint is to consider the state vector as an ontological entity, or a "real" physical entity. This viewpoint is supported by the novel WV-TSVF theory. An ingredient of this interpretational or theoretical viewpoint is to consider the state vector to be associated with an individual quantum system (at least in specific and well-defined experiments). Clearly, this viewpoint was questionable for the majority of physicists in the last century.

In this context, one should mention the counterintuitive Elitzur-Vaidman effect [55] (sometimes called "bomb-tester"), which concerns interaction-free measurements (IFM). This well known theoretical work revealed the ability to obtain information about an object's presence in a spatial region without ever "touching" it. Certainly, in the framework of classical physics, this is a paradox for the following reason: In a successful interaction-free "bomb detection", absolutely no known physical quantity, such as the energy, momentum, angular momentum, force, spin, etc., has been exchanged between the probe particle (e.g., a photon) and the object (the "bomb"). However, this physical information cannot be gained "at no costs" or "for free". The necessary "costs" are provided by the photon's wavefunction, following the principles of IFM. This predicted novel quantum effect has been experimentally verified and thus strongly suggests that the wavefunction is a real physical entity and not just an auxiliary quantity being used by the calculation of expectation values of the observables.

Moreover, the interpretation of the quantum wavefunction has recently become relevant to practical applications such as quantum cryptography and quantum computation; see the recent achievement to experimentally demonstrate the so-called "quantum supremacy" using GOOGLE's programmable quantum processor Sycamore with 53 qubits and executing a specific computational task [50]. Remarkably, therein the authors report a (very specific) quantum computational task being performed in about $200 \mathrm{~s}$, in contrast to the estimated 10,000 years needed by the (current existing) most powerful classical supercomputer to perform the same task. To stress this point, in simplified terms: The state vector calculated by the GOOGLE processor is a physical quantity that refers to one single quantum processor (i.e., one quantum system) and not to an ensemble of quantum processors.

In this context, a very recent (December 2019) quantum optical achievement [51] has been made, in which boson sampling, using 20 photons in a $10^{14}$-dimensional Hilbert space, is applied for demonstrating the quantum computational supremacy.

$(G)$ Supported by the above remarks and findings, we believe that the theory of WV and TSVF not only sheds new light on the issues concerning the interpretation of quantum mechanics, e.g., the time-inversion invariance of the Schrödinger equation and the meaning of quantum entanglement and non-local correlations, but further offers a new guide for our intuition to predict new effects as well as gives us a new (conceptual and practical) tool for designing new experiments and carry out specific measurements which may lead to the discovery of new quantum phenomena.

\section{Theoretical Part II: Quantum Information, Quantumness of Correlations and Quantum Thermodynamics}

\subsection{Introduction: Entanglement and Beyond}

Quantum entanglement (also called as entanglement for short), often also described as "spooky action at a distance" [56], is considered as the most characteristic feature of standard (non-relativistic) 
quantum mechanics [57, 58], since it has no analog in classical physics. Having no intuitive interpretation, in earlier times, it was usually considered to belong to "metaphysics" (and not to "real physics"). Entanglement violates the so-called Einstein's principle of locality. This led Einstein, Podolsky and Rosen (EPR) [57] to question the real nature of entanglement and conclude that the quantum description of physical reality cannot be complete. Therefore, certain (still unknown) "hidden parameters" remained to be found. However, the famous Bell inequalities [58] made it possible to experimentally test and falsify the EPR conclusion. In short, Einstein's principle of locality does not hold in general (see e.g. [20,49, 59]).

Remarkably, entanglement could only after about six decades find the entrance in physical and technological laboratories, initially as an information- theoretic resource, and very recently as a novel physical resource being as real as, e.g., energy $[16,59]$. The latter point is nowadays broadly accepted in quantum thermodynamics (QTD). However, being a very fragile quantity, entanglement is easily destroyed by environmental disturbances. This fact is considered to be the main reason preventing the realization of a quantum computer till now [16] (see, however, the very recent technological breakthroughs $[50,51]$ mentioned above).

In 2001, another discovery was made [17, 18]: "In-between" the sets of classical correlated states and those of the quantum entangled states, there exists a very large set of states exhibiting a new kind of quantum behavior; in this context, the notion "quantumness of correlations" (QoC) is often used. This novel phenomenon, as quantified, e.g., by quantum discord [17, 18] and various other measures of correlations [20], is also a rather general manifestation of non-classicality. Interestingly, these new kinds of correlations can be studied by locally measuring a specific part of a larger system (e.g., an $\mathrm{H}$ atom of a composite "H+environment" system) and in states in which entanglement (e.g., between the $\mathrm{H}$ atom and other adjacent particles) cannot exist.

Despite a considerable number of recent investigations studying QD and related quantifiers of the quantumness of correlations (see, e.g., [20]) it is a fact that these correlation effects remained far less understood until now than the well-studied quantum entanglement. Therefore, every new finding concerning the physical meaning and/or concrete applicability of QD (and the other related quantifiers) is of considerable scientific and technological relevance [60]. In particular, QD (and the related quantifiers) is considerably less fragile than entanglement and survives even at elevated temperatures in condensed systems. The experimental results of INS considered in the Experimental Part (see above) of this review article may contribute to the mentioned understanding of QD.

\subsection{Classical and Quantum Conditional Entropy}

In several subsections below, we mainly follow the introductory presentation [61] of Modi and Vedral; see also [62]. The work by Modi et al. [20] is a related review article containing the full theoretical details. An illustrative description of QD is given by Merali [60].

The exploration of general QoC beyond entanglement started in 2001 by observing that the definition of quantum conditional entropy is not unique. Firstly, consider the well-known Shannon entropy $[16,63]$

$$
H(X)=-\sum_{x} p_{x} \log p_{x}
$$


$X$ is a classical random variable which takes the values $x$ with probabilities $p_{x}$. The von Neumann entropy $[16,38]$ corresponding to a quantum system, $X$, in a quantum state, $\rho_{X}$, is given by

$$
S(X)=-\operatorname{Tr} \rho_{X} \log \rho_{X} .
$$

(Depending on the context, logarithms are often base two.)

Let $a$ and $b$ be random variables related to the states of the physical systems $A$ and $B$. The Bayes' rule of classical probability theory leads to the conditional entropy

$$
H(B \mid A) \equiv H(b \mid a)=H(a b)-H(a)
$$

which is a measure of our ignorance of $b$, under the condition that the information of $a$ has been given. Figure 7 illustrates these quantities.

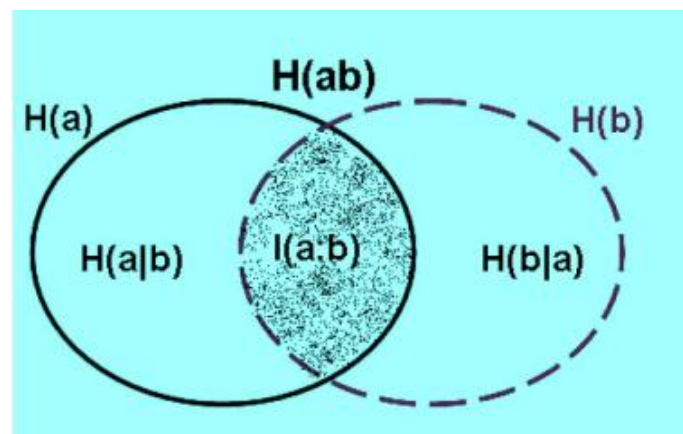

Figure 7 Schematic representation of conditional entropies, Eq. (56).

In the classical information theory, there is a fully equivalent way to express $H(b \mid a)$ : This is the ignorance of $b$ under the condition that $a$ is known to be in a specific $i$-th state $a_{i}$, the latter being weighted by the probability $p_{i}^{a}$ of this state to occur, i.e.,

$$
H(B \mid A) \equiv H(b \mid a)=\sum_{i} p_{i}^{a} H\left(b \mid a=a_{i}\right)
$$

However, as noted in $[17,18]$, the equivalence of Eqs. 56 and 57 breaks down in the quantum information theory, with the consequence that a new kind of quantum correlation ( such as QD) may appear [64]. Let us see how this may happen. The first expression of $H(b \mid a)$ is defined by the difference between the joint ignorance of $a$ and $b$ and that of $a$ alone. However, the second expression of $H(b \mid a)$, depends on the specific outcomes of the measurements on $a$. However, in clear contrast to the classical theory, measurement results in quantum mechanics depend on the basis of eigenvectors of the measured quantum observable. In addition, measurements, in general, change the quantum state of the measured system.

In quantum information theory, the aforementioned joint classical probability distributions are replaced with density operators and the von Neumann entropy replaces the classical Shannon entropy. Therefore, for the definition of the quantum conditional entropy, one has two possibilities: the first based on Eq. (56) and the second based on Eq. (57).

Let us first use Eq. (56) and define the quantum conditional entropy by the equation

$$
S^{(1)}(B \mid A)=S(A B)-S(A)
$$


as is usually done in quantum information theory [16]. Here is the following disturbing detail to note: $S^{(1)}(B \mid A)$ can be negative for entangled states. It took many efforts, and a long time, to give a physical meaning to this negativity.

The second possible definition of the quantum conditional entropy, based on Eq. (57), is by referring to the quantum measurements on system $A$ alone (and not on the whole system $A+B$ ), i.e., the joint state, $\rho^{A B}$, of $A+B$ is changed to

$$
\rho^{A B} \rightarrow \sum_{i} \Pi_{i}^{A} \rho^{A B} \Pi_{i}^{A}=\sum_{i} p_{i}|i\rangle\langle i| \otimes \rho_{i}^{B}
$$

Here, $\Pi_{i}$ is a positive- operator valued measure (POVM) [16] of rank one; $|i\rangle$ represents a classical flag being set on the measuring apparatus if the result $a_{i}$ is measured and $p_{i}=\operatorname{Tr}\left[\Pi_{i}^{A} \rho^{A B}\right]$ is the probability of obtaining the result $a_{i}$. As per the theory of standard quantum mechanics, in this case, the system $B$ goes over to the new state $\rho_{i}^{B}=\operatorname{Tr}_{A}\left[\Pi_{i}^{A} \rho^{A B}\right]$. This leads to the second definition of conditional entropy

$$
S^{(2)}(B \mid A)=S\left(B \mid\left\{\Pi_{i}\right\}\right)=\sum_{i} p_{i} S\left(\rho_{i}^{B}\right)
$$

Interestingly, it should be emphasized that the quantum conditional entropy defined in this way is always positive. However, as is well known, the initial state $\rho^{A B}$ in general changes after the measurement. It is also clear that, in general, $S^{(2)}(B \mid A) \neq S^{(2)}(A \mid B)$.

In addition, we may mention here that the physical interpretation of the negativity of the quantum conditional entropy in Eq. (60) was recently given a physical meaning with the aid of the quantuminformation procedure known as quantum state merging; see the review article by Modi et al. [20].

\subsection{Measures of Quantumness of Correlations}

\subsubsection{Quantum Discord}

Quantum discord can be defined as follows: First, one introduces the well-known quantity called mutual information (see e.g., [16])

$$
I(a: b)=H(a)+H(b)-H(a b)
$$

Making use of Eq. (57), one obtains an equivalent second expression for the classical mutual information:

$$
J(b \mid a)=H(b)-\sum_{i} p_{i}^{a} H\left(b \mid a=a_{i}\right)
$$

As can be seen easily, in general, this equivalence breaks down in quantum information theory:

$$
\delta(B \mid A) \equiv I(A: B)-J(B \mid A)
$$

as first observed by Ollivier and Zurek [17] who termed this difference as quantum discord (QD). Using the preceding equations, one easily obtains, for $Q D$, the following difference between the two expressions of conditional entropy:

$$
\delta(B \mid A)=S^{(2)}(B \mid A)-S^{(1)}(B \mid A)
$$


Moreover, and at the same time, an independent theoretical investigation by Henderson and Vedral [18] showed that the expression $J(B \mid A)$ essentially captures the classical correlations. More precisely, it was shown that $\max _{\left\{\Pi_{i}\right\}} J(B \mid A)$ represents the classical correlations. This further led to the physically motivated conclusion that $Q D$ is best defined as

$$
\delta(B \mid A)=\min _{\left\{\Pi_{i}\right\}}[I(A: B)-J(B \mid A)]
$$

We see that QD is asymmetric under the swap of the participating two subsystems, $A$ and $B$, which is also understandable on physical grounds.

\subsubsection{Measurement-Induced Disturbance}

The last expression for QD is based on a task very difficult to carry out in practice: the search for a minimum over a set of POVM's. In this context, recall that quantum measurements usually disturb the quantum system, in contrast to the classical measurements on classical systems. One can make the following (uncommon) remark: Classical states are not disturbed under quantum measurements. Based on these physical insights and observations, Luo introduced a new quantifier of QoC, focusing on the disturbance of a system through a measurement [65], which he called measurement-induced disturbance (MID). This measure is defined as the difference between the mutual information of a joint state, $\rho^{A B}$, and its dephased version, $\chi^{A B}$ :

$$
M I D=I\left(\rho^{A B}\right)-I\left(\chi^{A B}\right)=S\left(\rho^{A B}\right)-S\left(\chi^{A B}\right)
$$

The decoherence, (or canceling the non-diagonal elements of $\rho^{A B}$, or quantum dephasing, etc.) takes place on the marginal basis under consideration (which, of course, does not affect this basis).

It is trivially seen that all quantifiers of QoC vanish in the limiting case of the so-called classicalclassical (CC) states. These have the form

$$
\chi_{A B}=\sum_{a, b} p_{a b} \Pi_{a} \otimes \Pi_{b}
$$

Here, all projectors $\Pi_{i}$ correspond to completely distinguishable states, and therefore, $p_{a b}$ represent classical joint probabilities.

Furthermore, let us consider the well known separable states, also called quantum-quantum (QQ) states [20]

$$
\rho^{A B}=\sum_{i} p_{i} \rho_{i}^{A} \otimes \rho_{i}^{B}
$$

$\rho_{i}^{A}$ and $\rho_{i}^{B}$ are local states of the subsystems $A$ and $B$, respectively. The QQ states still contain quantum correlations, similar to how the two participating density operators do. As Luo discovered, the quantum correlations occurring (or being captured) in QQ-states offer a certain generalization of the concept of quantum entanglement [65]. The usefulness of MID and its interdependence with other measures of QoC (and in particular with QD) have been studied in previous investigations, see Refs. $[20,66]$.

The following important point can be easily verified: A state has zero QD and MID simultaneously. This physically indicates that in such a state, the conditional entropies in Eqs. (58) and (60) have the 
same value. This leads to the following new definitions: A state of this type, sometimes termed as a classical state, has the form

$$
\chi^{A B}=\sum_{i} p_{i}|i\rangle\langle i| \otimes \rho_{i}^{B}
$$

under the condition that measurements are made on system $A$ only. Equivalently, a classical state has the form $\chi^{A B}=\sum_{j} \rho_{j}^{A} \otimes p_{j}|j\rangle\langle j|$, under the condition that measurements are made on $B$ only. It is then easy to see that a fully "symmetric" classical state must have the aforementioned classical-classical (CC) form (67); obviously, any reference to measurement is here unnecessary.

Physically, one could say that MID captures the entropic costs of measurement in its marginal basis. In addition, it can be noticed that a generalized version of MID is related to the quantum work deficit within the framework of quantum Maxwell demon [67]; see below. This topic appears to play a central role in the interpretation (based on QTD) of the INS results presented in the Experimental Part.

\subsection{Measures based on Relative Entropy}

With respect to the classical states of the preceding section, another measure of QoC can be introduced based on the following intuition: This measure should represent a "distance", i.e., a term to be quantified, of a given state $\rho$ from the "nearest" classical state. In a similar way, one could formalize the distance of $\rho$ with another special property from the "nearest" state having another prescribed property. For example, the distance of an entangled state from a nearest separable state appears to be a physically meaningful measure of quantum entanglement.

A well known proper quantity formalizing the indicated distance between the states of the two systems $A$ and $B$ is the relative entropy (see e.g., [16])

$$
S(A \| B) \equiv-\operatorname{Tr}(A \log B)-S(A)
$$

where $S(A) \equiv-\operatorname{Tr}(A \log A)$ is the von Neumann entropy [38]. Interestingly, $S(A \| B)$ is always nonnegative and therefore captures the intuitive notion of a distance at least partially, despite its weakness of being a non-symmetric quantity with respect to the interchange $A \leftrightarrow B$.

So far, various specific distances using relative entropy have been introduced. This facilitates consistent comparison of different kinds of correlations, e.g., entanglement, QD, classical correlations, and also quantum dissonance. The latter is similar to QD, however, entanglement is excluded in this case.

In the following, is given a list of the different types of (quantum and classical) states mentioned in this Theoretical Part II, together with some short explanatory remarks.

First, we consider a state with no correlations at all, which should be a product state referring to an $N$-body system. It has the form

$$
\pi=\pi_{1} \otimes \ldots \otimes \pi_{N}
$$

where $\pi_{n}$ represents the $n$-th single subsystem. It is well known that a set of all product states, $\mathcal{P}$, is not convex, i.e., a mixture of product states may not necessarily belong to $\mathcal{P}$.

The set of classical states, $\mathcal{C}$, consists of a mixture of states that are locally distinguishable 


$$
\chi=\sum_{k_{n}} p_{k_{1} \ldots k_{N}}\left|k_{1} \ldots k_{N}\right\rangle\left\langle k_{1} \ldots k_{N}\left|=\sum_{\vec{k}} p_{\vec{k}}\right| \vec{k}\right\rangle\langle\vec{k}|
$$

This holds by definition. Here, one assumes that the set $\left\{\left|k_{n}\right\rangle\right\}$ span spans an orthonormal basis, and furthermore, that $p_{\vec{k}}$ represents a joint classical probability distribution. The correlations appearing in these states represent the correlations of classical physics [18]. Further, $\mathcal{C}$ is not a convex set; for instance, if two classical states are defined on different bases, their mixing can create a non-classical state.

We consider now the set of all separable states, denoted by $\mathcal{S}$, which contains mixtures of the form

$$
\sigma=\sum_{i} p_{i} \pi_{1}^{(i)} \otimes \ldots \otimes \pi_{N}^{(i)}
$$

In contrast to the previous cases, $\mathcal{S}$ is a convex set. The following physical property is important to be noted: Separable states can be created (constructed, prepared) using only local quantum operations and classical communication (LOCC) [16]. Nevertheless, they possess non-classical features in general $[17,18]$.

The following set-theoretical relations hold: The product states constitute a subset of the set of classical states; the latter constitutes a subset of the set of separable states. The set of entangled states and the set of separable states are disjoint. In addition, the set of entangled states is not convex.

Finally, we list here several distance-based measures of non-classical correlations, which commonly appear in the literature $[60,61]$ :

$$
\begin{aligned}
& E=\min _{\sigma \in \mathcal{S}} \boldsymbol{S}(\rho \| \sigma) \quad \text { (entanglement), } \\
& D=\min _{\chi \in \mathcal{C}} S(\rho \| \chi) \quad \text { (quantum discord), } \\
& Q=\min _{\chi \in \mathcal{C}} S(\sigma \| \chi) \quad \text { (quantum dissonance) }
\end{aligned}
$$

Furthermore, classical correlations can be quantified by the minimal distance of a given classically correlated state, $\chi$, from all product states, $\pi$, i.e.,

$$
C=\min _{\pi \in \mathcal{P}} S(\chi \| \pi)
$$

For full details about QoC and measures of non-classical correlations, see the review article by Modi et al. [20].

\subsection{Connecting Discord and Entanglement with Measurements and the Maxwell's Demon}

Let $A$ and $B$ be two interacting systems, one of which is being "observed" by (measured by, interacting with) a third quantum system (say, environment, or Maxwell's demon), $\mathcal{E}$. As already mentioned above, we will briefly consider investigations in which the measuring process affects the QoC appearing in the composite systems $A+B$ and $A+B+\mathcal{E}$.

\subsubsection{The Maxwell's Demon}


Maxwell's demon [68] is a well known theoretically conceived entity that is able to extract mechanical work from an "observed" or measured system that is in contact with a thermal bath. (Traditionally, the investigated system is mostly assumed to be in thermal equilibrium.) The process of interest is the possibility of extracting work by doing a measurement on a system. About 150 years ago, Maxwell [69] proposed that the powerful ability of a (fictitious) demon to determine the individual velocities and positions of the molecules of gas would imply the ability to extract mechanical work from the heat of the gas. (Essentially, this is achieved by separating fast moving molecules from the slow ones, with a technical procedure described in many textbooks.) This, or course, would break a fundamental physical axiom, i.e., the second law of (classical) thermodynamics.

This paradox has resisted resolution for over a century, and only recently was it resolved by Bennett [70] by extending the work done by Landauer [71]. In short, this work has clarified a highly non-trivial point: The demon's observation of the molecules produces physically relevant information (namely, the values of velocities/positions of the molecules) and this information must be stored in some other physical system, which is usually called the (demon's) memory. However, this memory cannot be infinite, and therefore, when the memory is full, this information must be erased. This erasure increases the entropy of the environment $[68,71]$.

Very recently, Yan et al. achieved the first experimental verification of Landauer's principle in a completely quantum system, in which both the bit to be erased and the heat reservoir had quantized energies [72]; see also [77].

\subsubsection{Quantum Deficit}

The measure of QoC, known as quantum deficit (or, quantum work deficit), has been motivated by the same questions as those in the preceding subsection, i.e., how would a physical mechanism of work extraction from quantum systems coupled to a thermal bath look like [73, 74]. Obviously, such questions involve links between the theory of QoC and, more generally, of QTD, with the corresponding entities of classical information theory and classical thermodynamics. Here, "classicality" is associated with the notion of the so-called LOCC [74]. [It may be noted that the concept of LOCC plays a significant role in the modern theory of quantum information and quantum computing [16].] To give more details, a system is considered to be classical (and/or contains only classical correlations) if and only if the amount of work that can be extracted from the total system (say, $W_{\text {tot }}$ ) is equal to the sum of works that can be extracted individually from each of the two subsystems (say, $W_{\text {sub }} \equiv W_{A}+W_{B}$ ), by also making use of the appropriate LOCC operations. These considerations have motivated the introduction of the new quantity, called quantum deficit, $\Delta$, by an associated "work deficit":

$$
\Delta \equiv W_{t o t}-W_{\text {sub }}
$$

Similar to the procedure carried out by von Neumann [38], Oppenheim et al., [73] postulated a relation between the work that can be extracted from the complete system $A B$ with the density operator, $\rho_{A B}$, and its quantum entropy, $S\left(\rho_{A B}\right)$ : 


$$
W_{t o t}=\log d_{A B}-S\left(\rho_{A B}\right)
$$

where $d_{A B}$ is the dimension of the Hilbert space of the system $A B$. [In theoretical works, it is a common practice to put the product of the Boltzmann constant with a temperature of the bath equal to 1 ; then the work is expressed in bits.] In essence, this equation indicates that more work can be extracted from purer states than from mixed ones; see the review article by Modi et al., [20].

\subsubsection{Connecting the Work Obtained by Maxwell's Demon with Quantum Discord}

In relation to the aforementioned considerations on Maxwell's demon, Zurek [75] proposed a similar measure for the optimized thermodynamical work extraction. In particular, he derived the following relation

$$
\Delta W=W_{Q}-W_{C}=k_{B} T D(A \mid B)
$$

where $T$ is the absolute temperature, $k_{B}$ : is the Boltzmann constant and $D$ : denotes QD. This relation connects QD (Eq. (75)) with the difference in the efficiency of quantum from that of a classical Maxwell's demon, with respect to their work extraction (not dissipation!) capabilities, denoted by $W_{Q}$ and $W_{C}$, respectively. $\Delta W$ is more or less equivalent to the quantum deficit, $\Delta$, introduced by Oppenheim et al. [20, 73]. A positive $W_{i}$ here implies that work is gained (extracted).

Based on this theoretical result, our striking experimental findings in the investigation of INS, presented in the Experimental Part (Section 2), can be understood in a straightforward manner; see subsection 4.8 , item $(D)$ below.

\subsubsection{Partial Quantum Measurements and Correlations}

Recently, extending the investigations discussed in the preceding subsections, Streltsov et al., [76] provided a more specific approach to QoC, which is based on the effect of a quantum measurement carried out on one subsystem of a composite system.

According to standard quantum mechanics [38], a strong measurement on a system $S$ in the quantum state $\rho^{S}$ creates entanglement (or other quantum correlations) between $S$ and the detection device (apparatus) $M$. For example, consider a von Neumann measurement of $S$ with using the density operator

$$
\rho^{S}=\sum_{i} p_{i}\left|i^{S}\right\rangle\left\langle i^{S}\right|
$$

with respect to the eigenbasis $\left\{\left|i^{S}\right\rangle\right\}$. According to the von Neumann theory of measurement [38], the final state of the composite system $S+M$ will be

$$
\rho_{\text {final }}=\sum_{i} p_{i}\left|i^{M}\right\rangle\left\langle i^{M}|\otimes| i^{S}\right\rangle\left\langle i^{S}\right|
$$

where $\left|i^{M}\right\rangle$ are the orthogonal states of the quantum system $M$. According to the preceding definitions, $\rho_{\text {final }}$ is then a CC state.

A more interesting situation appears when considering partial von Neumann measurements, i.e., measurements being restricted to dynamical variables of a subsystem $A$ of a larger composite system $S=A+B$. It is obvious that such measurements are common in real experimental situations; cf. 
Experimental Part. In this case, entanglement between $A$ and $M$ may might be created. Moreover, the close connection of this approach to the so-called one-way information deficit [20,66, 73] and QD was investigated. For instance, the authors proved the equivalence of one-way information deficit with the so-called minimal distillable entanglement [49] between $A+B$ and $M$. This kind of entanglement is due to the aforementioned partial measurement on $A$. In this process, QD appears to be equal to the associated minimal partial distillable entanglement; see [76] for details.

\subsubsection{On the Energetic Costs of a Quantum Measurement}

In the physical context under consideration in this article, there is a strong evidence that the energetic costs of a measurement is a fundamental aspect of the complete dynamics of the measurement and that it should have profound experimental consequences. Recently, Jacobs [78] emphasized that every quantum measurement has energetic costs, and therefore the measurement dynamics must be subject to the first law of thermodynamics. In other words, the energetic costs of measurement are intrinsically connected with the work value of the information acquired through the measurement.

This theoretical analysis also clarifies further aspects of the nature of Maxwell's demon. It is often tacitly assumed that measurement might even have vanishing energetic costs, despite the (since Landauer's work [71]) well-known fact that information does have an equivalent work value [20].

The main reason for the aforementioned tacit assumption is that, until recently, a possible energy exchange between the system and the demon observing it has been completely neglected. In addition, it is also assumed that only energy exchange takes place between the "system + demon" and the environment.

Furthermore, the aforementioned assumption of vanishing energetic costs of the measurement process simply overlooks, or denies, the costs paid by the measuring apparatus in order to perform a measurement [78]. Quantifying this issue, Jacobs showed that only in the unphysical case of the apparatus having access to a zero-temperature thermal bath, would the quantum measurement process be free of energetic costs [78]. More specifically, a given amount of information, say $\Delta I$, provided by a measuring apparatus, is accompanied by energy costs similar to those that a conventional heat engine must pay in order to produce an amount of work equivalent to $\Delta I$.

In the context of the experimental INS investigated in this article, these theoretical results should have considerable consequences in the interpretation of the striking experimental findings. In other words, in conventional theoretical treatments of the scattering results, the energetic costs of the measuring device are assumed to be zero, simply because the measuring device as well as the whole measurement dynamics are usually presumed to belong to the realm of classical mechanics. In clear contrast to this classical view, the energetic costs of quantum measurement are a real physical phenomenon, which one may consider to be intrinsically related to the first law of thermodynamics [78].

\subsection{Universality of Quantum Discord}


As is widely acknowledged, entanglement is a very fragile and/or ultra-short lived effect, which is easily destroyed by environmental influences [79]. Thus, it is natural to ask how robust and abundant $Q D$ and the related quantifiers are, and furthermore how entanglement could be a reason causing the striking findings in the experimental investigations of INS discussed in the Experimental Part above. Therefore, the recent investigations by Acín and his colleagues [80] are significant in this context, which revealed the remarkable fact considered in the following.

As exploited above, QD and other measures of QoC quantify non-classical correlations, including the vast realm beyond entanglement. Consequently, only states with vanishing QD exhibit strictly classical correlations. The following surprising, as well as a crucial, theorem, was proved by Ref. [80]: The set of purely classical states is extremely small. In fact, it is negligible with respect to the whole Hilbert space. In other words, a particular state picked out randomly from the set of all states of a system, has with probability equal to one (i.e., almost always) a positive QD. Moreover, starting from a state with zero discord, every arbitrarily small perturbation by the environment drives the system to a state with non-zero QD. This effect is quite significant for the INS context under investigation (Section 2).

These striking results have been found to hold for any Hilbert-space dimension. This obviously implies that they have immediate consequences for the foundations of quantum dynamics of open systems.

In the case of the INS experiments presented above, all materials represent open systems (in which the environment is also of quantum nature). The consequences of [80] for the interpretation of the observed momentum-transfer-deficit in the INS experiments are highly non-trivial: At "elevated" temperatures and based on the existing relatively "strong" $\mathrm{H}$-environment interactions, there is no entanglement. However, the phenomenon of QD (and/or further QoC aspects), might lead to the striking results of the experimental INS.

Recently, an insightful theoretical analysis of the local and non-local aspects of QD and other measures of QoC (and their relation to entanglement) has been presented by Agrawal et al. [81].

\subsection{Quantum Thermodynamics: Energetic Costs of Quantum Correlations}

Decoherence is the phenomenon that affects and/or destroys quantum correlation effects of all kinds (e.g., quantum-phase relations, quantum superpositions, quantum interference, entanglement, etc.), and leads to the "appearance of a classical world in quantum theory" [79]. Decoherence is an irreversible quantum phenomenon, i.e., it breaks the time-inversion invariance of the Schrödinger's equation and thus may define and/or introduce a "direction of the time arrow" in physical theories. A considerable number of theoretical models of decoherence, based on a large variety of physical motivations and/or intuitions, have been proposed; see, e.g., the monograph [79] and the vast literature cited therein. Furthermore, it plays a significant role in quantum information theory and processing as well as in the huge efforts put in the development of a quantum computer and the related quantum technologies [16]. Indeed, decoherence is the main reason that has thus far prevented the successful production of a "sufficiently good performing" quantum processor. Only 
very recently was an associated breakthrough $[50,51]$ in this quantum technology field been achieved; cf. related remarks in subsection 3.4 , item $(F)$, above.

\subsubsection{Erasure of Quantum Correlations - A theoretical Model and Simulation}

A theoretical model by Schulman and Gaveau [82, 83], which is independent of the general theories of decoherence, provides additional evidence about the energetic costs of manipulating quantum correlations.

In this model, elastic collisions between two quantum oscillators, $A$ and $B$, are theoretically studied. The free Hamiltonians of the two systems are $H_{A}$ and $H_{B}$, and the collisions are described by a time-dependent impulsive interaction, $V_{A B}$. Hence the total Hamiltonian is

$$
H=H_{A}+H_{B}+V_{A B}
$$

The theoretical investigation starts with the assumption that prior to the collision, $A$ and $B$ are not entangled, which implies that the complete density matrix, $\rho(0)$, can be a product, i.e., $\rho(0)=$ $\rho_{A}(0) \otimes \rho_{B}(0)$. The collision happens at time $t=0 \pm \epsilon$, with $0<\epsilon \ll 1$.

At a later time, $t>\epsilon$, the system of the two oscillators has the density operator

$$
\rho(t)=U(t) \rho(0) U^{\dagger}(t)
$$

Here, $U(t)$ is the evolution operator corresponding to the full Hamiltonian $H$. After the collision, the particles become non-interacting once again but are now entangled and $\rho(t)$ is not the product state of the two reduced density operators $\rho_{A}(t)=\operatorname{Tr}_{B} \rho(t)$ and $\rho_{B}(t)=\operatorname{Tr}_{A} \rho(t)$, which one usually attributes to the two oscillators. Thus, in general, the two particles become quantum correlated due to their collision.

Furthermore, one commonly assumes that when the particles are separated after the collision, the quantum correlations (or entanglement) can be simply dropped, as all physical quantities of each of the two particles cannot be dependent on any correlation between them. [One recognizes here the so-called Einstein locality principle, i.e., the belief about physical reality, which led to the famous Einstein-Podolsky-Rosen (EPR) paradox [57].] Consequently, one has the replacement

$$
\rho(t) \rightarrow \rho_{A}(t) \otimes \rho_{B}(t)
$$

in which the quantum correlations are irreversibly erased "by hand", since the exact operator $U(t)$ is unitary and thus causes a reversible time evolution only. This replacement should not affect the physical properties of either $A$ or $B$ at time $t>0$, in which $V_{A B}(t)=0$ holds.

The numerical results of Ref. [82] contradict this intuitive expectation (the intuition being based on classical mechanics). For specific interaction Hamiltonians, putting

$$
\Delta \rho(t)=\rho_{A}(t) \otimes \rho_{B}(t)-\rho(t)
$$

Schulman and Gaveau derived [83] the relation for sufficiently short times:

$$
\Delta E \equiv \operatorname{Tr}(\Delta \rho(t) H)=\operatorname{Tr}\left(\Delta \rho(t) V_{A B}\right)>0
$$

That is, the replacement of the exact $\rho(t)$ by the approximative and non-entangled state $\rho_{A}(t) \otimes$ $\rho_{B}(t)$, which, as explained, is tantamount to the erasure of quantum correlations, necessarily 
increases the energy of the two-oscillator system. Hence, one could conclude that this result is erroneous since the basic energy conservation law of physics is violated. Of course, this is a highly paradoxical result, as the authors nicely put it: "...losing quantum correlations should not heat the gas. You do not burn your fingers because of a partial trace over a density matrix" [82].

Although it does not hold universally, this theoretical result was derived from a broad class of Hamiltonians [83]. This detail sheds more light on the considered effect. In order to show this, numerical simulations using two different Hamiltonians were compared. The first Hamiltonian is given by the spin-boson model of two quantum oscillators

$$
H=H_{A}+H_{B}+V_{S B}=\omega_{a} a^{\dagger} a+\omega_{b} b^{\dagger} b+g\left(a^{\dagger}+a\right)\left(b^{\dagger}+b\right)
$$

Here, the simulations confirmed the presence of the effect under consideration. In contrast, in the simulations using the well known Jaynes-Cummings Hamiltonian (see, e.g. [42])

$$
H=H_{A}+H_{B}+V_{J C}=\omega_{a} a^{\dagger} a+\omega_{b} b^{\dagger} b+g\left(a^{\dagger} b+b^{\dagger} a\right)
$$

the effect (87) just disappeared and one obtained the intuitively expected result $\Delta E=0$ [82].

The following point is important here. In the Jaynes-Cummings model, the typical quantum contributions $a^{\dagger} b^{\dagger}$ and $a b$, which naively seem to correspond to the "violation of energy conservation", are fully ignored. These terms are known to capture the typical short-lived quantum fluctuations, and as the simulations showed, they are the origin of the surprising effect of Eq. (87).

Putting this observation in more general terms, the authors emphasized that here one has a timedependent interaction, $V_{A B}(t)$, meaning that the considered system is an open quantum system, and hence energy conservation does not apply strictly [82]. Moreover, the following physical argument might hold here [82]: The additional energy $\Delta E>0$ is provided by the translational degrees of freedom of $A$ and $B$, which are not included explicitly in the model Hamiltonian. Thus, they could be formally considered to represent an "effective bath" to which $A$ and $B$ are coupled.

In more illustrative terms, one could say that Maxwell's demon (cf. subsection 4.5.1) is "observing" this time-dependent collisional process of $A$ and $B$, and by "consuming" the collision-created quantum correlations (85) he succeeds to "produce" additional mechanical work (here, the vibrational energies of the oscillators). Of course, these remarks are in full accordance with the spirit of Landauer's Principle: "Information is physical" [71].

\subsubsection{Quantum Heating and Quantum Cooling}

As has been shown in the preceding subsection, the theoretical model of Ref. [82] enforces the erasure of quantum correlations (85) in the simulation "by hand", i.e., after each collision, the created correlations are canceled arbitrarily. However, this does not include the erasure mechanism explicitly. A detailed theoretical model addressing this mechanism was proposed by Kurizki and collaborators $[84,85]$. These studies explore the destruction of correlations (equivalently, decoherence) in a more general physical context of quantum cooling and/or heating of a two-level system. Certain unexpected non-Markovian cooling/heating effects were shown to be caused by the novel aspects of coherent quantum dynamics of the system-bath interaction in time intervals significantly shorter than the characteristic memory time of the bath's quantum dynamics. This theoretical result emphasizes 
the significance of the bath being a quantum system and participating actively in the collisional dynamics, and thus is in full agreement with the analogous interpretation of the "anomalous" INS results presented in the Experimental Part.

A result analogous to Eq. (87) was derived, first for brief quantum non-demolition (QND) measurements [84] and then for the general case of any abrupt disturbance [85].

The analysis by Ref. [84] considers the QND measurement of a quantum system, $S$, by a second quantum system, $D$, i.e., a detector. The interaction Hamiltonian is time-dependent, $H_{S D}(t)$. The and the total "system + bath" Hamiltonian is

$$
H_{t o t}=H_{S}+H_{B}+H_{S B}
$$

where $H_{S}$ : is the free Hamiltonian of the system, $H_{B}$ is the Hamiltonian of the bath, and $H_{S B}$ : is the Hamiltonian of the system-bath interaction. Furthermore, the coupling of $H_{\text {tot }}$ with the detector $D$ yields

$$
H(t)=H_{t o t}+H_{S D}(t)
$$

Consider the evolution of the entire system in an ultra-short time interval. Here, it is crucial that the two coupling Hamiltonians $H_{S B}$ and $H_{S D}$ are not simplified in order to conform to the popular rotating-wave approximation of quantum optics; see e.g., [42]. In other words, strict energy conservation between the system and the bath or the detector is not imposed [84], similar to the model described in the preceding subsection. It was shown that an impulsive disturbance of a system by a detector always increases the system's energy (which the authors called "heating") immediately after the measurement [84]. This derived theoretical result was confirmed by the accompanying numerical simulations carried out using a sufficiently short-time disturbance.

To give more details, for $0 \leq t \leq \tau$, Kurizki et al., derived the following results:

$$
\begin{aligned}
\left\langle H_{S B}(0)\right\rangle \leq & \rightarrow\left\langle H_{S B}(\tau)\right\rangle=0 \\
\left\langle H_{S D}(t)\right\rangle & =-\left\langle H_{S B}(t)\right\rangle \\
\left\langle H_{D}\right\rangle & =\text { const }
\end{aligned}
$$

The energy transfers described by these relations are interconnected with the associated changes in the amount of entanglement of the system and the bath, which cause a redistribution of energy and entropy between the three parts of the considered complete system [84].

To facilitate understanding, further details of this process could be helpful. Consider sufficiently short times, $t=\tau+\Delta t$ after the measurement. In this case, $H_{S D}(t \geq \tau)=0$ holds, the rotating wave approximation does not apply and consequently, $\left\langle H_{S}+H_{B}\right\rangle$ is not conserved, although $\left\langle H_{\text {tot }}\right\rangle$ is conserved. $\left\langle H_{S B}\right\rangle$ decreases toward its undisturbed value and thus one obtains the results

$$
\begin{aligned}
\left.\frac{d}{d t}\left(\left\langle H_{S}+H_{B}\right\rangle\right)\right\rfloor_{\tau+\Delta t} & >0 \\
\left.\frac{d}{d t}\left\langle H_{S B}\right\rangle\right\rfloor_{\tau+\Delta t} & <0
\end{aligned}
$$

This is equivalent to a quantum heating of the "system + bath" [84], which takes place immediately after the disturbance. [Furthermore, a little later, the inverse effect was shown to occur, i.e., a 
quantum cooling was predicted, a result being of potential relevance for experimental investigations of quantum gas condensates.]

Referring to the experimental results of INS that are under consideration in this article, it may be noted that the neutron- $\mathrm{H}$ scattering process is by no means a QND measurement. Therefore, it is important to notice that the above results have been extended to the general case of any arbitrary abrupt disturbance [85]. This latter case then becomes applicable in the context of the experimental INS process investigated in this article, where the neutron-H impulsive collision does represent such an abrupt disturbance. In the example of subsection 2.6, the neutron- $\mathrm{H}$ collision corresponds to the abrupt measurement process (with the neutron corresponding to $D$ ), in which the system-bath $\left(\mathrm{H}_{2}\right.$ nanotube) interaction dynamics leads to a quantum heating (here, an anomalous increase of the translational energy of the $\mathrm{H}_{2}$ molecule). In this process, the quantum environment (i.e., the Cnanotube fragment adjacent to the $\mathrm{H}_{2}$ molecule) is participating actively.

[Of course, the associated small quantum heating of the C-nanotube fragment, which interacts with the $\mathrm{H}_{2}$ molecule, cannot be measured with the experimental set-up of Ref. [35].]

4.7.3 Thermodynamic Meaning of Negative Conditional Entropy and the Generalized Landauer's Principle

In the discussions of QoC presented in the preceding subsections, the concept of conditional (information-theoretic) entropy was of central importance. In their work, del Rio et al. [19], analyzed the standard implications of the "classical" Landauer's principle [71] and arrived at the conclusion that they no longer remain valid if quantum information is present. In the "quantum" case, the main finding is as follows: The work cost of information erasure is determined by the quantum entropy of the system that is conditioned on the quantum information (of the system), which is available to the observer (or to an observing demon). The more an observer knows about the system, the less are the (energetic) costs of the erasure under consideration. As one can easily see, this result emphasizes the significance of the conditional entropy of information. The relevance of the latter within the theoretical framework of QoC was already discussed above.

In formal terms, the main result of del Rio et al. [19] could be described as follows: Consider a system, $A$, being correlated with a quantum memory, $Q$. The work that an observer (or demon), having access to $Q$, requires to do in order to erase the information content of $A$, is denoted by $W(A \mid Q)$. In the thermodynamic limit, which here means that the observer has access to many identical copies of $A$ and erases them jointly, the authors constructed a specific erasure procedure which has work costs no larger than

$$
W(A \mid Q)=k_{B} T \ln (2) S(A \mid Q)
$$

per single copy of $A$ [19]. $S(A \mid Q)$ is the well known von Neumann conditional entropy [20], (cf. Eq. (58))

$$
S(A \mid Q)=S(A Q)-S(Q)
$$


Furthermore, assuming the validity of Landauer's principle for classical observers (demons), these work costs are shown to be optimal.

However, for a quantum Maxwell's demon, the situation may change significantly. For instance, as the previous equation shows, the work cost for erasure could be negative. I.e., if the observer has access to $Q$, the erasure process can even produce work (instead of consuming work).

This is rather easily shown with the aid of the following simple example: Let the combined system $A+Q$ be a closed system in a pure quantum state. Thus, its von Neumann entropy is zero,

$$
S(A Q)=0
$$

However, the reduced state of $Q$ is mixed, having a positive entropy $S(Q)>0$. Hence, Eq. (96) yields $S(A \mid Q)<0$, and therefore Eq. (95) implies negative work costs,

$$
S(A \mid Q)<0 \Rightarrow W(A \mid Q)<0
$$

which of course is tantamount to energy gain.

The "strange" negative conditional entropy obtained in this result has a thermodynamic operational meaning. This interpretation also extends the earlier investigations dealing only with the information- theoretic interpretations of this negative value of entropy [19, 20].

Furthermore, as the authors of [19] noticed, this result shows that QD can properly quantify the difference between the erasure-costs by using a quantum or classical memory $Q$.

It should be emphasized that these results do not violate the second law of thermodynamics. In other words, the proposed process of erasure is not cyclic, and the negative work costs correspond to the consumption of entanglement between the two quantum systems $A$ and $Q$. More importantly, if one wants to construct a cyclic process, this consumed entanglement can only be restored with the aid of another process, which has positive work costs. Thus, it can be concluded that the overall work costs of a cyclic process will be non-negative; see Addendum of Ref. [19].

\subsubsection{QoC in the Quantum Dynamics of INS}

After the preceding general considerations of QoC and their significance in the context of INS, it may be helpful to summarize, and reconsider, the time-dependent $\mathrm{QoC}$ accompanying the collisional process somewhat more formally.

Due to its many-body character, the $\mathrm{H}-\mathcal{E}$ interaction cannot lead to a pure state for $\mathrm{H}$ (and another one for $\mathcal{E}$ ). Thus, let $\rho_{\lambda}^{H}$ (with $\lambda$ indicating the possible available states) be one of the mixed $\mathrm{H}$-states and $\rho_{\lambda}^{n}$ be the associated mixed neutron-state (after the impulsive collision). Due to the aforementioned $\mathrm{H}-\mathcal{E}$ interactions, which are by no means negligible in the considered experiments,

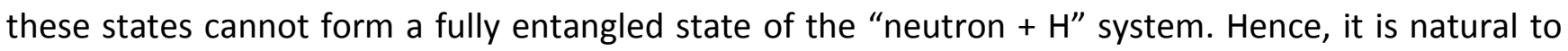
assume the existence of a quantum discordant state, cf. Eq. (68), i.e.,

$$
\rho^{n, H}\left(t \sim \tau_{\text {exp }}\right) \approx \int d \lambda p(\lambda) \rho^{n}(\lambda) \otimes \rho^{H}(\lambda)
$$

Here, $\tau_{\text {exp }}>0$ is the neutron-H scattering time window, which is very short (roughly in the pico- to femto-second range) due to the Fermi pseudo-potential of the delta-function form and which creates the neutron- $\mathrm{H}$ quantum correlations (in some cases, perhaps also a very short-lived neutron- $\mathrm{H}$ 
entanglement) described by $\rho^{n, H}$. In illustrative terms, one may say that the environment $\mathcal{E}$, and/or an associated Maxwell's demon, "observes" the neutron-H system continuously, thus continuously changing the amount of correlations between the neutron and the $\mathrm{H}$ atom.

During the characteristic relaxation time denoted by $\tau_{\text {relax }}$, which is a characteristic quantity of the material at a given temperature and pressure, the complete 3-body system "neutron $+\mathrm{H}+\mathcal{E}$ " will be strongly correlated, i.e., for times $\tau_{\text {exp }}<t \sim \tau_{\text {relax }}$, it will be in the state $\rho^{n, H, \varepsilon}$.

At longer times, $t \gg \tau_{\text {relax }}$, the neutron will be far away and the excited scattering system

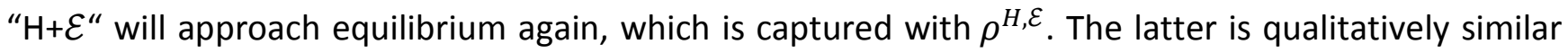
to the quantum correlated state of " $\mathrm{H}+\mathcal{E}$ " before the disturbing collision with the neutron. At the same time, the neutron should occupy a mixed state $\rho^{n}$.

Furthermore, assuming an ideal high-resolution detector for measuring momentum and according to the standard reduction postulate of quantum mechanics in relation to a measurement, the actual detection of the neutron will produce an (at least approximately) pure state, $\left|\mathbf{k}_{1}^{\theta}\right\rangle\left\langle\mathbf{k}_{1}^{\theta}\right|$. This is the same as the assumption of the conventional theory, i.e., the conventional plane wave approximation of the standard theory $[1,27]$.

In summary, we could represent the correlations in the dynamics of the INS investigated in this work with the aid of the following steps (see also [14]):

$$
\begin{aligned}
t=-\infty & : \rho^{n} \otimes \rho^{H, \mathcal{E}} \\
\text { collision: } t \approx 0 & : \rho^{n, H} \otimes \rho^{\varepsilon} \\
0 \approx t \leq \tau_{\text {exp }} \sim 10^{-14} \mathrm{~S} & :\left(\int d \lambda p(\lambda) \rho^{n}(\lambda) \otimes \rho^{H}(\lambda)\right) \otimes \rho^{\varepsilon} \\
\text { excited complete system: } \tau_{\text {exp }}<t \sim \tau_{\text {relax }} & : \rho^{n, H, \mathcal{E}} \\
t \gg \tau_{\text {relax }} & : \rho^{H, \mathcal{E}} \otimes \rho^{n} \\
\text { detector measures } n \text { at } t=+\infty & : \rho^{H, \mathcal{E}} \otimes\left|\mathbf{k}_{1}^{\theta}\right\rangle\left\langle\mathbf{k}_{1}^{\theta}\right|
\end{aligned}
$$

(For simplifying the notation, the $t$-variable is not shown explicitly in a time-dependent quantity, such as $\rho^{H, \mathcal{E}}$, appearing in several lines.)

Obviously, the amount of QD and of any other measure of quantum correlations (cf. preceding subsections) that are relevant in the INS process, changes with time. For example, as time goes on, the QD between any two of the three parts of the "neutron $+\mathrm{H}+\mathcal{E}$ " system is erased and/or created continuously.

4.7.5 Connection with the Experimental Results of INS - Excess E-Transfer and the Number of "Consumed" Qubits

A connection between the theoretical frameworks described above and the INS results described in the Experimental Part can be made, especially with the striking "anomaly" under consideration. To be specific, we first consider the results from the $\mathrm{H}_{2} \mathrm{O}$-beryl material, Figure 4 . The stretching vibrational peak is centered at $K_{v} \approx 11 \AA^{-1}$ and $E_{v} \approx 470 \mathrm{meV}$. One sees that the conventional $E$ transfer at this $K$-transfer should be $E_{\text {conv }} \approx 250 \mathrm{meV}$, which is the $E$-value of the $\mathrm{H}$-recoil parabola at the momentum transfer $K_{v}$. Thus, the non-conventional $E$-surplus is $\Delta E_{v} \approx 220 \mathrm{meV}$. Using these 
numerical values (and $T=7 \mathrm{~K}$ ) and by applying the generalized Landauer's principle, Eq. (95), we obtain a rough estimate of the qubit-number associated with the observed "anomaly" as

$$
\mathrm{H}_{2} \mathrm{O} \text { in Beryl: } \quad \text { \#Qubits } \sim \frac{\Delta E_{v}}{k_{B} T \ln (2)} \sim 530
$$

The same considerations are now applied on the roto-translational data of the $\mathrm{H}_{2}$-nanotube system (Figure 5 , at $T=23 \mathrm{~K}$ ). For example, at the chosen wavevector $K \equiv K_{t r} \approx 6 \AA^{-1}$ and the associated non-conventional $E$-surplus, $\Delta E_{t r} \approx 30 \mathrm{meV}$ which is obtained from the figure via visual inspection, we obtain the numerical estimate

$$
\mathrm{H}_{2} \text { in C-nanotube: \#Qubits } \sim \frac{\Delta E_{t r}}{k_{B} T \ln (2)} \sim 22
$$

Of course, these rough estimates are based on the assumption that the quantum Maxwell's demon operates (or acts) "with full efficiency" on the $\mathcal{E}$ system, i.e., he extracts the mentioned amount of "anomalous" surplus work (or excess $E$-transfer) "optimally", which he then "transfers" to the $\mathrm{H}$ atom.

In view of these different numbers of qubits, it may be helpful to notice the large difference between the interaction energies of the struck $H$ atom with $\mathcal{E}$ in these two examples: The $H$ atom from the $\mathrm{OH}$-bond of $\mathrm{H}_{2} \mathrm{O}$ is subjected to a strong intramolecular interaction (in addition to the much weaker interaction with the surface of the beryl-channel), whereas the translating $\mathrm{H}_{2}$ molecule interacts only weakly with the surface of the C-nanotube. Hence, the quantum Maxwell's demon has a larger number of qubits to his disposition in the first case than that in the second one.

These estimates of qubit numbers are based on the (arbitrary) assumption that no additional classical bits are "consumed" in the considered process. Recall that classical bits are associated with positive costs of erasure. Hence, it would be better to consider the estimated numbers in the relations (101) and (102) as representing the estimated lower bounds of the qubit-numbers (being consumed with negative work costs).

Furthermore, it should also be noted that there is a significant difference between the two corresponding momentum transfers, a fact that prevents a further insightful (or physically sensible) comparative analysis of the estimated qubit numbers.

\subsection{Additional Remarks II}

Modern QTD and the associated theory of QoC in interacting systems succeed in providing a qualitative interpretation (and an intuitive understanding) of the new experimental INS effects presented in the "Experimental Part" above.

(A) Although quantum entanglement between $\mathrm{H}$ atoms and/or $\mathrm{H}_{2}$ molecules is most likely not present in condensed systems at finite temperatures and for finite time intervals, such as in conditions related to the INS experiments under consideration, QD and other QoC-measures can be certainly expected to be present. In this context, recall the aforementioned wide abundance of QD [80] and its robustness (as compared to entanglement). This opens up a new research path and reveals the possibility of introducing various theoretical methods of quantum correlation in the investigations in physics, chemistry, molecular biology, and various material sciences. 
$(B)$ The INS effect under consideration in this article provides strong evidence for the quantum nature of the environment $\mathcal{E}$ interacting with the $\mathrm{H}$ atom that is colliding with the neutron and also for the significance of $\mathcal{E}$ for the whole scattering process. Here, recall the ultra-fast non-Markovian dynamics of the quantum bath (or $\mathcal{E}$ ), which was shown to be essential for the new quantum heating effect captured in Eqs. (94).

$(C)$ Here, it should be emphasized again that the neutron- $\mathrm{H}$ scattering under consideration is mainly incoherent, i.e., a single neutron collides with a single $\mathrm{H}$ atom. [Clearly, the usual multiple scattering effects observed in several scattering experiments are irrelevant in the present analysis.] In addition, when the scattering $\mathrm{H}$ atom is partially "bounded" to a few adjacent particles, the impinging neutron should scatter from an object which has an effective mass higher than 1 a.m.u. of a single free $\mathrm{H}$ atom. More importantly, and obviously, this binding, according to the conventional theory, can never lead to a decrease in the effective mass of the $\mathrm{H}$ atom, simply because the adjacent particles cause a resistance (or hindrance) to the free motion of the $\mathrm{H}$ atom (or the $\mathrm{H}_{2}$ molecule).

In view of these physical explanations, it is not surprising that those who believe that conventional theory is strictly valid must dismiss the "anomalous" effect of the momentum- transfer deficit, simply because this effect would imply that, as some proponents of the conventional theory put it, "... conventional theories of neutron scattering, quantum mechanics, and energy conservations are invalid"; see [86]. However, such criticisms are fully based on the common belief that INS is appropriately described as a two-particle collisional process, with a (classical) environment playing only a minor (and passive) role, and therefore they just evaporate in view of the preceding point $(B)$, i.e., the quantum dynamical nature of $\mathcal{E}$.

$(D)$ Further connections of QoC and QTD with the INS experimental results are briefly mentioned here:

(D1) Based on Zurek's relation, Eq. (80) and the physical considerations of the preceding subsections, one can extract an estimated value of the $\mathrm{QD}, D(H \mid \mathcal{E})$, associated with the "anomalous" excess $E$-transfer observed in the experiment. In other words, one identifies the non-conventional part of the measured E-transfer with the "superior ability" of the quantum Maxwell's demon over the classical one in extracting work from the correlations.

(D2) The theoretical result of Schulman and Gaveau, Eq. (87), which connects the nonconventional excess energy, $\Delta E$, to the erased (or consumed) quantum correlations, may be used to roughly estimate the expectation value of the interaction Hamiltonian, $V_{H \mathcal{E}}$. In doing so, one associates the two systems $A$ and $B$ with $\mathrm{H}$ and $\mathcal{E}$, which is a physical assumption and/or working hypothesis.

(D3) A similar conceptual association can be also obtained by identifying Kurizki's ultra-fast quantum heating effect, Eqs. (94), with the observed "anomalous" excess $E$-transfer in INS.

(D4) Extending the remarks and discussions of the preceding subsection 4.7.5 and making use of the generalized Landauer's principle by del Rio et al., Eq. (95), one can estimate a value for the quantum conditional entropy $S(H \mid \mathcal{E})$, which has turned out to be negative here. This further supports the physical relevance of the work by del Rio et al. [19] and the offered physical meaning of negative conditional entropy in the context of QTD and experimental INS. 


\section{Applicability of the Two Theoretical Frameworks of WV-TSVF and QoC-QTD - A Comparison}

In connection with the theoretical part describing the WV-TSVF framework, a particularly important point should be highlighted:

The analysis based on QoC does not specify the "anomalous" deviations from the conventional INS theory (see Experimental Part) as a momentum-transfer deficit. Rather, it predicts an "energy transfer surplus" at non-specified given values of momentum transfer. Indeed, this is an alternative possibility for describing the non-conventional theoretical behavior of INS observed in the experiments, because in this case, one can also equivalently capture the observations with the aid of a reduced effective mass of the scattering particle.

It should be noted that the general theory of QoC and QTD primarily deals with energy and with other quantities originating from classical thermodynamics (e.g., entropy or free energy). In other words, so far, the theoretical framework of QTD has not been generalized to deal with other physical quantities, such as momentum, originating from mechanics. However, a quantum Maxwell's demon could, in principle, enforce both "anomalous features" (i.e., momentum and energy transfers) under consideration.

Extending these remarks, an additional experimental indication is observed related to the comparison between both the theoretical frameworks of WV-TSVF and QoC-QTD investigated in this article, which should be briefly discussed here. The INS experiments on the vibrational modes of $\mathrm{H}_{2} \mathrm{O}$ molecules, described in subsection 2.5, show an additional specific feature, namely, a conventional energy transfer, equal to the excitation energy for the $\mathrm{OH}$-stretching vibrational modes, which are well known from IR and Raman spectroscopy (see textbooks), and which is rather well localized in the $K-E$ plane (see Figure 4). Therefore, so to speak, one observes in this experiment an associated anomalously reduced $\hbar K$ related to a given (and "normal") $E$-value of the stretching vibrational modes. Remarkably, this corresponds exactly to the formulation and predictions of the WV-TSVF theoretical model (Section 3), which considers the physical quantity of momentum (and not energy) directly.

In addition, recall that the measured roto-translational excitation of $\mathrm{H}_{2}$ in $\mathrm{C}$-nanotubes (subsection $2.6)$ is not well localized in the $K-E$ plane and thus, the observed "anomalous scattering" may be related to both the quantities $\hbar K$ and $E$. at the same time. However, so far, we are not aware of any INS (or neutron Compton scattering, or deep inelastic neutron scattering) experiment that could have succinctly shown a "conventionally expected" value of the momentum transfer, $\hbar K$, in association with an "anomalously" increased energy transfer $E$.

Hence, on comparing the two theoretical frameworks considered in this article, and at least in the present context of the experimental INS, the WV-TSVF theory appears to be applicable in a more straightforward manner. On the other hand, the potential (even though rather speculative) perspective for making direct contact with quantum information theory, e.g., by attributing an experimentally determined number of qubits to the observed "anomalous" effect (see subsection 4.7.5), make QoC and QTD an attractive theoretical tool to be used in further explorations.

\section{Further Considerations and Discussion}


1. Various well known quantum effects of hydrogen, being attributed to its light mass, appear in physical and chemical processes. Such effects are, e.g., quantum tunneling, quantum delocalization as well as the widely known effect of zero-point motion of an $\mathrm{H}$ atom in a molecular bond. Such quantum effects are nowadays also considered in quantum-chemical calculations of $\mathrm{H}$-containing materials since they are also of technological importance. For example, these quantum effects affect the dissociative adsorption of the $\mathrm{H}_{2}$ molecule on solid surfaces and also the diffusion of $\mathrm{H}$ atoms on surfaces. Therefore, they are of potential relevance to the catalytic properties of those surfaces. Furthermore, these quantum effects could also be observed in the field of molecular biology, because they affect the strength and structure of hydrogen bonds, including those in DNA, proteins, and in biomolecular interactions and bonding.

The above-mentioned examples represent one-body quantum effects and have therefore not been considered in this review article, which deals with quantum effects between two or more nonidentical quantum objects.

2. As already mentioned in subsection 2.7 of the Experimental Part, the novel theoretical framework of WV-TSVF appears to provide a new experimental method to extract the width of the final state of the $\mathrm{H}$ atom (momentum-space wavefunction of the struck $\mathrm{H}$ atom) from the measured momentum-transfer deficit. In other words, the novel WV-TSVF theory allows us to conceive a method for extracting new information from the neutron-H collisions using the current existing instrumentation.

3. With the widely used quantum-chemical computations, spectral peak positions in terms of energy can be calculated satisfactorily. However, as far as we know, until now there exist no quantum-chemical programs that are capable of calculating the two-dimensional $K-E$-spectra, i.e., not only the $E$-, but also the $K$-positions of the "peaks". The technological advances offered by the recently developed two-dimensional spectrometers (like ARCS, Oak Ridge Nat. Lab.) make the need for the creation of such quantum chemical programs obvious.

4. As already mentioned in subsection 3.4, point (C), the two dynamical observables $\hat{q}$ and $\hat{P}$ occurring in the interaction Hamiltonian of Eq. (39) refer to two different systems, the neutron and the atom. Hence, as Vaidman [45] pointed out, the concept of WV captures the interferences of a quantum entangled wave. In other words, quantum correlation effects appear naturally in the novel WV-TSVF theory as well, which of course, should be not surprising at all.

Since the precise interrelation between the two theoretical frameworks under consideration has so far remained largely unexplored, it is hoped that the INS experiments investigated in this review article could contribute to further related theoretical developments.

5. The presented findings of the experimental INS support the following conclusion: The conventional INS theory should be amended by including the concepts of WV and TSVF theory, theoretical concepts of quantum information (such as QoC), and general QTD.

6. Both theoretical frameworks addressed in the Theoretical Parts of this paper and the recent INS experiments presented therein, strongly support the scientific content by Landauer's (and Bennett's; see above) widely known aphorism: "Information is physical" [87]. Furthermore, our results also provide considerable evidence for the relevance and usefulness of the quantum information theory in the fields of natural and applied sciences (physics, chemistry, etc.), which is nowadays beginning to be 
appreciated by theorists, experimenters, and also quantum-technology engineers. Hence, we strongly feel that the theoretical frameworks discussed in this article must receive more attention in the education of these natural sciences and also in engineering.

7. With respect to the significance of the experimental results described above, and in particular, in view of the observations of the momentum-transfer deficit and the associated effective mass reduction of the $\mathrm{H}$ atom, it may be appropriate to briefly mention a few reasonable speculative ideas here about the technological importance of the new quantum theoretical advances discussed in this article. For example:

(i) The mobility of $\mathrm{H}^{+}$concernsis related to the efficiency of fuel cells (hydrogen technology).

(ii) Dynamical effects of the host-hydrogen interactions in industrial hydrogen-storage materials may affect the performance of such materials [88].

(iii) The mobility of $\mathrm{Li}^{+}$is essential for "electro-mobility" (e.g., batteries, cars).

In view of the contents of this article, the quantum environment of the particle of interest should play an essential role in these examples. Furthermore, for the improvement of technology, not only are the energetics (as described by the common quantum-chemical calculations of the multi-dimensional Born-Oppenheimer energy-landscapes) of the particles in these materials crucial but so are the timedependent dynamics of their momenta.

8. The intensity deficit effect. In this review article, another "anomalous" neutron scattering effect, appearing at higher initial energies of the neutron (say, $E_{0}=1-100 \mathrm{eV}$ ), has not been considered. This is the so-called "intensity deficit", a striking deficit in the measured scattering intensity of hydrogen; see [89-91] and references therein. Its physical origin was attributed to decoherence (erasure of quantum correlations) of quantum entanglement in an ultra-short time window of the neutron-proton collision [92, 93] - at that time, the authors were not aware of investigations on QD. A fully analogous intensity deficit effect was predicted and observed in the quasi-elastic (Compton-like) electron-H scattering from $\mathrm{H}_{2}$ and $\mathrm{HD}$ in the gas phase by Cooper et al. [94]. Needless to say, these findings also did not find an interpretation of the observed results within the conventional electron scattering theory.

At present, the intensity deficit effect is under investigation within the theoretical framework of WV and TSVF, especially in connection with the imaginary part $[6,39]$ of a WV capturing the intensity of scattering.

9. In view of the successful qualitative interpretations of the reported INS experiments using both the theoretical frameworks under consideration, it seems natural to believe (or at least to suspect) that a deep theoretical connection between WV-TSVF and QoC-QTD must exist, which remains to be discovered and explored.

\section{Author Contributions}

The author did all work.

\section{Competing Interests}

The author has declared that no competing interests exist. 


\section{References}

1. Squires GL. Introduction to the theory of thermal neutron scattering. 2nd ed. Cambridge: Cambridge University Press; 2012.

2. Aharonov $\mathrm{Y}$, Rohrlich D. Quantum paradoxes - quantum theory for the perplexed. Weinheim: Wiley-VCH; 2008.

3. Aharonov $\mathrm{Y}$, Bergmann PG, Lebowitz JL. Time symmetry in the quantum process of measurement. Phys Rev. 1964; 134: B1410.

4. Aharonov $Y$, Albert DZ, Vaidman L. How the result of a measurement of a component of the spin of a spin-1/2 particle can turn out to be 100. Phys Rev Lett. 1988; 60: 1351.

5. Aharonov $Y$, Vaidman L. Properties of a quantum system during the time interval between two measurements. Phys Rev A. 1990; 41: 11.

6. Tamir B, Cohen E. Introduction to weak measurements and weak values. Quanta. 2013; 2: 7-17.

7. Aharonov $\mathrm{Y}$, Cohen $\mathrm{E}$, Elitzur AC. Foundations and applications of weak quantum measurements. Phys Rev A. 2014; 89: 052105.

8. Aharonov $\mathrm{Y}$, Cohen $\mathrm{E}$, Waegell $\mathrm{M}$, Elitzur AC. The weak reality that makes quantum phenomena more natural: Novel insights and experiments. Entropy. 2018; 20: 854.

9. Shikano Y. Theory of "Weak Value" and quantum mechanical measurements, in: Measurements in quantum mechanics. Reza Pahlavani M (Ed.) Shanghai and Rijeka: InTech; 2012.

10. Ritchie NWM, Story JG, Hulet RG. Realization of a measurement of a "Weak Value". Phys Rev Lett. 1991; 66: 1107.

11. Lundeen JS, Sutherland B, Patel A, Stewart C, Bamber C. Direct measurement of the quantum wavefunction. Nature. 2011; 474: 188-191.

12. Dressel J, Malik M, Miatto FM, Jordan AN, Boyd RW. Colloquium: Understanding quantum weak values: Basics and applications. Rev Mod Phys. 2014; 86: 307.

13. Chatzidimitriou-Dreismann CA. Weak measurement and two-state-vector formalism: Deficit of momentum transfer in scattering processes. Quanta. 2016; 5: 61-84.

14. Chatzidimitriou-Dreismann CA. Weak measurement and weak values - New insights and effects in reflectivity and scattering processes, J Phys Conf Ser. 2018; 1071: 012007.

15. Chatzidimitriou-Dreismann CA. Weak values and two-state-vector formalism in elementary scattering and reflectivity - A new effect. Universe. 2019; 5: 58.

16. Nielsen MA, Chuang IL. Quantum computation and quantum information. 2nd ed. Cambridge: Cambridge University Press; 2010.

17. Ollivier $\mathrm{H}$, Zurek WH. Quantum discord: A measure of the quantumness of correlations. Phys Rev Lett. 2001; 88: 017901.

18. Henderson L, Vedral V. Classical, quantum and total correlations. J Phys A Math Gen. 2001; 34: 6899.

19. del Rio L, Aberg J, Renner R, Dahlsten O, Vedral V. The thermodynamic meaning of negative entropy. Nature. 2011; 474: 61

20. Modi K, Brodutch A, Cable H, Paterek T, Vedral V. The classical-quantum boundary for correlations: Discord and related measures. Rev Mod Phys. 2012; 84: 1655. 
21. Chatzidimitriou-Dreismann CA. Quantumness of correlations and Maxwell's demon in molecular excitations created by neutron scattering. Int J Quantum Chem. 2015; 115: 909-929.

22. Feynman RP, Leighton RB, Sands M. The Feynman lectures on physics. Vol. III, Quantum Mechanics. Reading USA: Addison-Wesley; 1965.

23. Mitchell PCH, Parker SF, Ramirez-Cuesta AJ, Tomkinson J. Vibrational Spectroscopy with Neutrons. New Jersey: World Scientific; 2005.

24. Kearley GJ, Johnson MR. Vibrational spectroscopy with neutrons - Where are we now? Vib. Spectrosc. 2010; 53: 54-59.

25. Diallo SO, Azuah RT, Abernathy DL, Rota R, Boronat J, Glyde HR. Bose-Einstein condensation in liquid He near the liquid-solid transition line. Phys Rev B. 2012; 85: 140505.

26. Wide Angular-Range Chopper Spectrometer ARCS, SNS, Oak Ridge National Laboratorry, USA. Available from: https://neutrons.ornl.gov/arcs/

27 Watson GI. Neutron Compton scattering. J Phys Condens Matter. 1996; 8: 5955.

28. Tietje IC. Neutron Compton scattering-critical analysis of some basic theoretical assumptions. J Phy Conf Ser. 2012; 380: 012014.

29. Chopper spectrometer MARI, ISIS Neutron and Muon Source, Science and Technology Facilities Council, UK. Available from: https://www.isis.stfc.ac.uk/Pages/mari.aspx

30. Kearley GJ, Fillaux F, Baron MH, Bennington S, Tomkinson J. A new look at proton transfer dynamics along the hydrogen bonds in amides and peptides. Science. 1994; 264: 1285.

31. Anovitz LM, Mamontov E, Ben Ishai P, Kolesnikov Al. Anisotropic dynamics of water ultraconfined in macroscopically oriented channels of single-crystal beryl: A multifrequency analysis. Phys Rev $E$. 2013; 88: 052306.

32. Fine-Resolution Fermi Chopper Spectrometer SEQUOIA, SNS, Oak Ridge National Laboratorry, USA. Available from: https://neutrons.ornl.gov/sequoia/

33. Kolesnikov Al, Anovitz LM, Mamontov E, Podlesnyak A, Ehlers G. Strong anisotropic dynamics of ultra-confined water. J Phys Chem B. 2014; 118: 13414.

34. Spencer EC, Huang B, Parker SF, Kolesnikov AI, Ross NL, Brian F, et al. The thermodynamic properties of hydrated $\mathrm{p}-\mathrm{Al}_{2} \mathrm{O}_{3}$ nanoparticles. J Chem Phys. 2013; 139: 244705.

35. Olsen RJ, Beckner M, Stone MB, Pfeifer P, Wexler C, Taub H. Quantum excitation spectrum of hydrogen adsorbed in nanoporous carbons observed by inelastic neutron scattering. Carbon. 2013; 58: 46.

36. Indirect geometry spectrometer TOSCA, ISIS Neutron and Muon Source, Science and Technology Facilities Council, UK. Available from: https://www.isis.stfc.ac.uk/Pages/tosca.aspx

37. Georgiev PA, DK.Ross, De Monte A, Montaretto-Marullo U, Edwards RAH, Ramirez-Cuesta AJ et al. In situ inelastic neutron scattering studies of the rotational and translational dynamics of molecular hydrogen adsorbed in single-wall carbon nanotubes (SWNTs). Carbon. 2005; 43: 895.

38. Von Neumann J. Mathematical foundations of quantum mechanics. Princeton: Princeton University Press; 1955.

39. Jozsa R. Complex weak values in quantum measurement. Phys Rev A. 2007; 76: 044103.

40. Pati AK, Wu J. Conditions for anomalous weak value. ArXiv. 2014; arXiv:1410.5221 [quant-ph] 
41. Aharonov Y, Botero A, Nussinov S, Popescu S, Tollaksen J, Vaidman L. The classical limit of quantum optics: Not what it seems at first sight. New J Phys. 2013; 15: 093006.

42. Scully MO, Zubairy MS. Quantum optics. Cambridge: Cambridge University Press; 1997.

43. Aharonov $Y$, Cohen E, Ben-Moshe S. Unusual interactions of pre-and-post-selected particles. EPJ Web of Conf. 2014; 70: 00053.

44. Messiah A. Quantum mechanics. Vol. II. Amsterdam: North Holland Publ. Comp.; 1965.

45. Vaidman L. Comment on "How the result of a single coin toss can turn out to be 100 heads". ArXiv. 2014; ArXiv:1409.5386 [quant-ph]

46. van Hove L. Correlations in space and time and Born approximation scattering in systems of interacting particles. Phys Rev. 1954; 95: 249-262.

47. Ferrie C, Combes J. How the result of a single coin toss can turn out to be 100 heads. Phys Rev Lett. 2014; 113: 120404.

48. Romito A, Jordan AN, Aharonov Y, Gefen Y. Weak values are quantum: You can bet on it. Quantum St: Math Found. 2016; 3: 1.

49. Horodecki R, Horodecki P, Horodecki M, Horodecki K. Quantum entanglement. Rev Mod Phys. 2009; 81: 865.

50. Arute F, Arya K, Babbush R, Bacon D, Bardin JC, Barends R, et al. Quantum supremacy using a programmable superconducting processor. Nature 2019; 574: 505-510.

51. Wang H, Qin J, Ding X, Chen MC, Chen S, You X, He YM, et al. Boson sampling with 20 input photons and a 60-Mode interferometer in a 1014-dimensional Hilbert space. Phys Rev Lett. 2019; 123: 250503.

52. Arora S, Barak B. Computational complexity - a modern approach. Cambridge: Cambridge University Press; 2009.

53. Fortnow L. The status of the P versus NP problem. Communications of the ACM. 2009; 52: 78-86.

54. Papadimitriou CH. Computational Complexity. Reading: Addison-Wesley; 1995.

55. Elitzur AC, Vaidman L. Quantum mechanical interaction-free measurements. Found Phys. 1993; 23: 987-997.

56. Schrödinger E. Die gegenwärtige Situation in der Quantenmechanik. Die Naturwissenschaften. 1935; 23: 807 and 823 and 844.

57. Einstein A, Podolsky B, Rosen N. Can quantum-mechanical description of physical reality be considered complete? Phys Rev. 1935; 47: 777.

58. Bell JS. On the Einstein Podolsky Rosen paradox. Physics. 1964; 1: 195.

59. Vedral V. Quantum entanglement. Nat Phys. 2014; 10: 256.

60. Merali Z. Quantum computing: The power of discord. Nature. 2011; 474: 24-25.

61. Modi K, Vedral V. Unification of quantum and classical correlations and quantumness measures. AIP Conf Proc. 2011; 1384: 69.

62. Modi K, Paterek T, Son W, Vedral V, Williamson M. Unified view of quantum and classical correlations. Phys Rev Lett. 2010; 104: 080501.

63. Shannon CE, Weaver W. The mathematical theory of communication. Urbana: University of Illinois; 1949. 
64. Datta A. Studies on the role of entanglement in mixed-state quantum computation. NY: Cornell University; 2008.

65. Luo S. Using measurement-induced disturbance to characterize correlations as classical or quantum. Phys Rev A. 2008; 77: 022301.

66. Lang MD, Caves CM, Shaji A. Entropic measures of non-classical correlations. Int J Quantum Inform. 2011; 9: 1553.

67. Brodutch A, Terno DR. Quantum discord, local operations, and Maxwell's demons. Phys Rev A. 2010; 81: 062103.

68. Maruyama K, Nori F, Vedral V. Colloquium: The physics of Maxwell's demon and information. Rev Mod Phys. 2009; 81: 1.

69. Maxwell JC. Theory of heat. London: Appleton; 1871.

70. Bennett $\mathrm{CH}$. The thermodynamics of computation-A review. Int J Theor Phys. 1982; 21: 905.

71. R. Landauer R. Irreversibility and heat generation in the computing process. IBM J Res Dev. 1961; 5: 183.

72. Yan LL, Xiong TP, Rehan K, Zhou F, Liang DF, Chen L, et al. Single-Atom demonstration of the quantum Landauer principle. Phys Rev Lett. 2018; 120: 210601.

73. Oppenheim J, Horodecki M, Horodecki P, Horodecki R. Thermodynamical approach to quantifying quantum correlations. Phys Rev Lett. 2002; 89: 180402.

74. Horodecki M, Horodecki K, Horodecki P, Horodecki R, Oppenheim J, Sen A, et al. Local information as a resource in distributed quantum systems. Phys Rev Lett. 2003; 90: 100402.

75. Zurek WH. Quantum discord and Maxwell's demons. Phys Rev A. 2003; 67: 012320.

76. Streltsov A, Kampermann H, Bruß D. Linking quantum discord to entanglement in a measurement. Phys Rev Lett. 2011; 106: 160401.

77. Silva JPP, Sarthour RS, Souza AM, De Oliveira IS, et al. Experimental demonstration of information to energy conversion in a quantum system at the Landauer limit. ArXiv. 2014; arXiv: 1412.6490v3 [quant-ph].

78. Jacobs K. Quantum measurement and the first law of thermodynamics: The energy cost of measurement is the work value of the acquired information. Phys Rev E. 2012; 86: 040106(R).

79. Schlosshauer M. Decoherence and the quantum-to-classical transition. Berlin: Springer; 2007.

80. Ferraro A, Aolita L, Cavalcanti D, Cucchietti FM, Acin A. Almost all quantum states have nonclassical correlations. Phys Rev A. 2010; 81: 052318.

81. Agrawal P, Sazim S, Chakrabarty I, Pati AK. Local, nonlocal quantumness and information theoretic measures. Int J Quantum Inf. 2016; 14: 1640034.

82. Schulman LS, Gaveau B. Ratcheting up energy by means of measurement. Phys Rev Lett. 2006; 97 : 240405.

83. Gaveau B, Schulman LS. Energetic consequences of decoherence at small times for coupled systems. J Phys A Math Theor. 2010; 43: 055308.

84. Erez N, Gordon G, Nest M, Kurizki G. Thermodynamic control by frequent quantum measurements. Nature. 2008; 452: 724-727.

85. Gordon G, Bensky G, Gelbwaser-Klimovsky D, Rao DB, Erez N, Kurizki G. Cooling down quantum bits on ultrashort time scales. New J Phys. 2009; 11: 123025. 
86. Mayers J, Reiter G. Spurious indications of energetic consequences of decoherence at short times for scattering from open quantum systems. AIP Adv. 2012; 2: 032137.

87. Landauer R. Information is physical. Physics Today. 1991; 44: 23-29.

88. Ramirez-Cuesta AJ, Jones MO, David WIF. Neutron scattering and hydrogen storage. Mater Today. 2009; 12: 54-61.

89. Chatzidimitriou-Dreismann CA, Abdul-Redah T, Streffer RMF, Mayers J. Anomalous deep inelastic neutron scattering from liquid $\mathrm{H}_{2} \mathrm{O}-\mathrm{D}_{2} \mathrm{O}$ : Evidence of nuclear quantum entanglement. Phys Rev Lett. 1997; 79: 2839.

90. Chatzidimitriou-Dreismann CA, Abdul-Redah T, Kolarić B. Entanglement of protons in organic molecules: An attosecond neutron scattering study of $\mathrm{C}-\mathrm{H}$ bond breaking. J Am Chem Soc. 2001; 123: 11945-11951.

91. Chatzidimitriou-Dreismann CA, Vos M, Kleiner C, Abdul-Redah T. Comparison of electron and neutron compton scattering from entangled protons in a solid polymer. Phys Rev Lett. 2003; 91 : 057403.

92. Chatzidimitriou-Dreismann CA, Stenholm S. On correlation approach to scattering in the decoherence timescale-Towards the theoretical interpretation of NCS and ECS experimental findings. ArXiv. 2007; arXiv: quant-ph/0702038v1.

93. Chatzidimitriou-Dreismann CA. Attosecond protonic quantum entanglement in collision experiments with neutrons and electrons. Laser Physics. 2005; 15: 780-788.

94. Cooper G, Hitchcock AP, Chatzidimitriou-Dreismann CA. Anomalous quasielastic electron scattering from single $\mathrm{H}_{2}, \mathrm{D}_{2}$, and $\mathrm{HD}$ molecules at large momentum transfer: Indications of nuclear spin effects. Phys Rev Lett. 2008; 100: 043204.

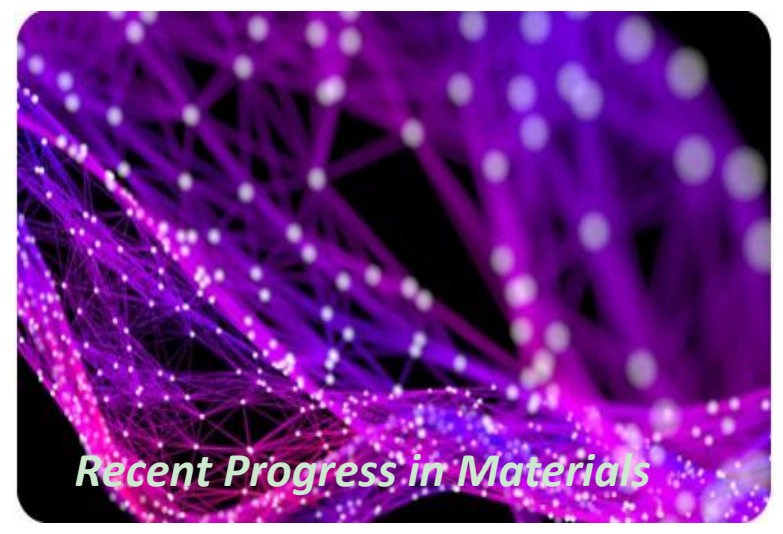

Enjoy Recent Progress in Materials by:

1. Submitting a manuscript

2. Joining in volunteer reviewer bank

3. Joining Editorial Board

4. Guest editing a special issue

For more details, please visit: http://www.lidsen.com/journals/rpm 